\title{
Orientational Dynamics of a Functionalized Alkyl Planar Monolayer Probed by Polarization-selective Angle-resolved Infrared Pump-probe Spectroscopy
}

\author{
Jun Nishida, Chang Yan, and Michael D. Fayer* \\ Department of Chemistry, Stanford University, Stanford, CA, 94305 \\ E-mail: fayer@stanford.edu Phone: (650) 723-4446
}

\section{Supporting Information}

\section{A. Local Field Corrections for IRLD and PSAR-PP Experiment}

In this section, we will discuss the macroscopic/microscopic local field corrections necessary to interpret IRLD and PSAR-PP data correctly. The local field corrections have been developed by Shen and Heinz to study orientations of interfacial molecules by sum-frequency generation (SFG) spectroscopy. Shen and Heinz calculated a radiation field from an infinitely thin polarization sheet and derived necessary local field correction factors. ${ }^{1,2}$ Yamaguchi and Tahara calculated local field correction factors for linear reflection (LR) spectroscopy. ${ }^{3}$ The approach by Yamaguchi and Tahara can be easily extended to understand the local field effects in IRLD and PSAR-PP experiments.

While particularly in SFG experiments signals emitted from nonlinear polarizations are often homodyne-detected, in IRLD/PSAR-PP experiments the signals are heterodyned by the field which is transmitted through the substrate. Because the transmitted field, acting as a local oscillator interfering with the signal, is different for $s$ - and $p$-polarizations, this difference must be taken into account to compare the observed signal levels.

In the following subsections, we will first discuss the local field effect for IRLD experiment in detail. The application of the local field effects to PASR-PP experiment is straightforward once they are formulated for IRLD.

\section{A1. Macroscopic Local Field Effect}

Our goal in this subsection is to derive the dichroic ratio in Eq. (S.12) with the effective incident angle $\chi^{\prime}$ and the $p / s$ enhancement factor $a_{p / s}$ given in Eqs. (S.10a) and (S.10b), which takes the macroscopic local field effect into account.

Strictly speaking, the sample studied here should be treated as a five-layer system with the air, the rhenium head group layer, the alkyl chain layer, the $\mathrm{SiO}_{2}$ layer and the $\mathrm{CaF}_{2}$ substrate. In the following discussion, we will combine the last three layers and treat them as a one layer with the refractive index $n_{b}=1.399$, which is the refractive index for $\mathrm{CaF}_{2}$ at $5 \mu \mathrm{m}$. This is justified by (1) that the refractive indices for the $\mathrm{C}_{11}$ alkyl chain $(\sim 1.5),{ }^{4} \mathrm{SiO}_{2}(\sim 1.35),{ }^{5}$ and $\mathrm{CaF}_{2}$ are relatively well index-matched, and (2) that $\mathrm{C}_{11}$ alkyl chain layer $(\sim 1 \mathrm{~nm})$ and $\mathrm{SiO}_{2}$ layer $(\sim 100 \mathrm{~nm})$ are too thin compared with the wavelength $(\sim 5 \mu \mathrm{m})$ to modulate the phase of the beam, and therefore no 


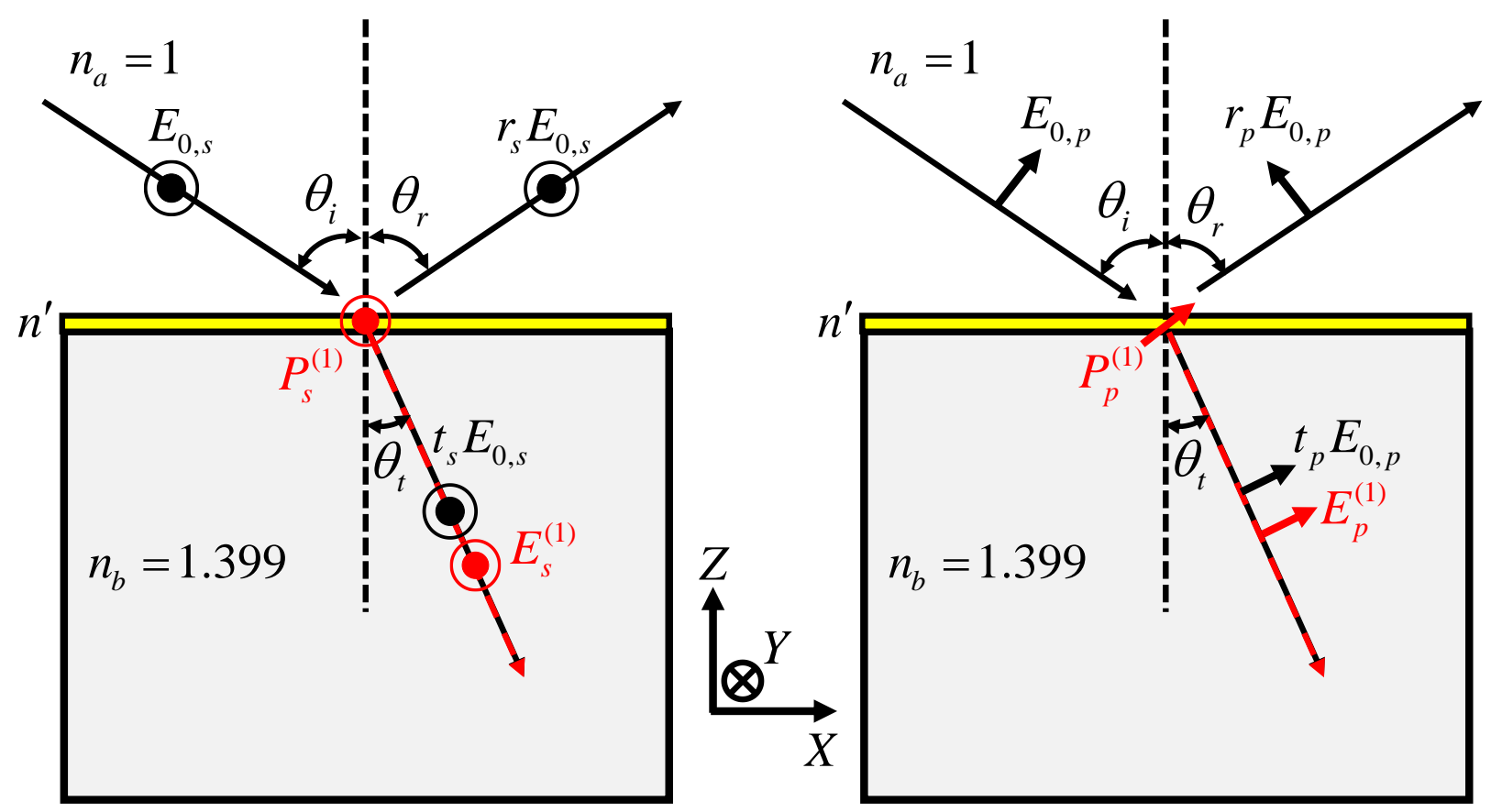

Figure S1. Infrared absorption experiment on an interfacial layer for $s$-polarized (left) and $p$-polarized (right) infrared beams. The infrared beam is incident from the air and induces a first-order polarization $P^{(1)}$, which emits a signal field $E^{(1)}$. $E^{(1)}$ interferes with a transmitted field to be heterodyned-detected.

additional reflection as a source of the macroscopic local field correction will be introduced by the existence of these layers. Under this approximation, the sample can be regarded as a threelayer system as studied by LR/SFG works.

Figure $\mathrm{S} 1$ shows the beam configuration for the infrared absorption spectroscopy for the $s$ - and $p$-polarized beams. Note that the incident angle $\theta_{i}$, the reflection angle $\theta_{r}=\theta_{i}$, the transmission angle $\theta_{t}=\sin ^{-1}\left(n_{a} \sin \theta_{i} / n_{b}\right)$ are independent of the polarization. In each geometry, the incident beam induces a first-order polarization from the infinitely thin sheet of absorbers at the surface (polarization sheet), which emits a first-order field. The emitted field propagates collinearly with the transmitted field.

Our interest here is what the overall transmitted fields are in each geometry. By solving the Maxwell equation with proper boundary conditions, ${ }^{3}$ the overall transmitted fields in each geometry are obtained as

$$
\begin{aligned}
& E_{t, s}=t_{s} E_{0, s}+\frac{i \omega}{2 c \varepsilon_{0} n_{a} \cos \theta_{i}} L_{Y Y} P_{Y}^{(1)^{\prime}} \\
& E_{t, p}=t_{p} E_{0, p}+\frac{i \omega}{2 c \varepsilon_{0} n_{a} \cos \theta_{i}}\left[\frac{\cos \theta_{i}}{\cos \theta_{t}} L_{X X} P_{X}^{(1)^{\prime}} \cos \theta_{i}+\frac{n_{a}}{n_{b}} L_{Z Z} P_{Z}^{(1)^{\prime}} \sin \theta_{i}\right]
\end{aligned}
$$

where $t_{s}$ and $t_{p}$ are the Fresnel transmission coefficients from the air to the substrate given by 


$$
\begin{aligned}
& t_{s}=\frac{2 n_{a} \cos \theta_{i}}{n_{a} \cos \theta_{i}+n_{b} \cos \theta_{t}} \\
& t_{p}=\frac{2 n_{a} \cos \theta_{i}}{n_{a} \cos \theta_{t}+n_{b} \cos \theta_{i}}
\end{aligned}
$$

$\vec{L}$ is a tensorial local field correction factor given by

$$
\begin{aligned}
L_{X X} & =\frac{2 n_{a} \cos \theta_{t}}{n_{a} \cos \theta_{t}+n_{b} \cos \theta_{i}} \\
L_{Y Y} & =\frac{2 n_{a} \cos \theta_{i}}{n_{a} \cos \theta_{i}+n_{b} \cos \theta_{t}} \\
L_{Z Z} & =\frac{2 n_{b} \cos \theta_{i}}{n_{a} \cos \theta_{t}+n_{b} \cos \theta_{i}}\left(\frac{n_{a}}{n^{\prime}}\right)^{2}
\end{aligned}
$$

and $\vec{P}^{(1)^{\prime}}$ is an interfacial polarization induced by the incident field through a first-order susceptibility $\vec{\chi}^{(1)}$ as

$$
\begin{aligned}
& P_{X}^{(1)^{\prime}}=\varepsilon_{0} \chi_{X X}^{(1)} L_{X X} \cos \theta_{i} E_{0, p} \\
& P_{Y}^{(1)^{\prime}}=\varepsilon_{0} \chi_{Y Y}^{(1)} L_{Y Y} E_{0, s} \\
& P_{Z}^{(1)^{\prime}}=\varepsilon_{0} \chi_{Z Z}^{(1)} L_{Z Z} \sin \theta_{i} E_{0, p}
\end{aligned}
$$

Note that, as shown by Shen and Heinz, ${ }^{1,2}$ the polarization is induced by the local field incident from the air, and thus the local field correction factor $\vec{L}$ appears in Eqs. (S.4a)-(S.4c).

The first terms in Eqs. (S.1a) and (S.1b), $t_{s} E_{0, s}$ and $t_{p} E_{0, p}$, are the transmitted field in the absence of the polarization sheet, which serves as the local oscillators. The second terms are the first-order signal fields emitted by the first-order polarizations:

$$
\begin{aligned}
E_{s}^{(1)} & =\frac{i \omega}{2 c \varepsilon_{0} n_{a} \cos \theta_{i}} L_{Y Y} P_{Y}^{\prime}=\frac{i \omega}{2 c n_{a} \cos \theta_{i}} L_{Y Y}^{2} \chi_{Y Y}^{(1)} E_{0, s} \\
E_{p}^{(1)} & =\frac{i \omega}{2 c \varepsilon_{0} n_{a} \cos \theta_{i}}\left[\frac{\cos \theta_{i}}{\cos \theta_{t}} L_{X X} P_{X}^{(1)^{\prime}} \cos \theta_{i}+\frac{n_{a}}{n_{b}} L_{Z Z} P_{Z}^{(1)^{\prime}} \sin \theta_{i}\right] \\
& =\frac{i \omega}{2 c n_{a} \cos \theta_{i}}\left[\frac{\cos \theta_{i}}{\cos \theta_{t}} \chi_{X X}^{(1)} L_{X X}^{2} \cos ^{2} \theta_{i}+\frac{n_{a}}{n_{b}} \chi_{Z Z}^{(1)} L_{Z Z}^{2} \sin ^{2} \theta_{i}\right] E_{0, p},
\end{aligned}
$$

where the interfacial polarization was substituted with Eqs. (S.4a)-(S.4c). 
When the time-averaged infrared absorption spectra as in Figure 1B in the main text were acquired, the IR intensity passing thorough the $\mathrm{SiO}_{2} / \mathrm{CaF}_{2}$ substrate without the functionalized monolayer was measured and used as a background intensity $\left(I_{\mathrm{bg}}\right)$, and then the $\mathrm{SiO}_{2} / \mathrm{CaF}_{2}$ substrate with the functionalized monolayer was installed instead and the transmitted intensity was recorded ( $\left.I_{\text {sig }}\right)$. The absorbance was derived as

$$
A=-\log _{10}\left(\frac{I_{\text {sig }}}{I_{\mathrm{bg}}}\right)
$$

Thus for the $s$-polarized beam, the absorbance is calculated as

$$
\begin{aligned}
A_{s}=-\log _{10}\left(\frac{\left|t_{s} E_{0, s}+E_{s}^{(1)}\right|^{2}}{\left|t_{s} E_{0, s}\right|^{2}}\right) & \approx-\log _{10}\left(1+2 \operatorname{Re}\left[t_{s} E_{0, s} E_{s}^{(1)} /\left|t_{s} E_{0, s}\right|^{2}\right]\right) \\
& \approx-\frac{2}{\ln (10)} \cdot \operatorname{Re}\left[t_{s} E_{0, s} E_{s}^{(1)} /\left|t_{s} E_{0, s}\right|^{2}\right] \\
& =\frac{2}{\ln (10)} \cdot \frac{\omega}{2 c n_{a} \cos \theta_{i}} \frac{L_{Y Y}^{2}}{t_{s}} \operatorname{Im}\left[\chi_{Y Y}^{(1)}\right]
\end{aligned}
$$

As an approximation, the fact that the signal field $E_{s}^{(1)}$ is much smaller than the local oscillator $t_{s} E_{0, s}$ was used. Note that $\vec{\chi}^{(1)}$ is defined such that the absorptive part is the imaginary part. Similarly, for the $p$-polarized beam, the absorbance is derived as

$$
A_{p}=\frac{2}{\ln (10)} \cdot \frac{\omega}{2 c n_{a} \cos \theta_{i}} \frac{1}{t_{p}}\left[\frac{\cos ^{3} \theta_{i}}{\cos \theta_{t}} L_{X X}^{2} \operatorname{Im}\left[\chi_{X X}^{(1)}\right]+\frac{n_{a}}{n_{b}} \sin ^{2} \theta_{i} L_{Z Z}^{2} \operatorname{Im}\left[\chi_{Z Z}^{(1)}\right]\right]
$$

Therefore, the observed dichroic ratio $\rho \equiv A_{p} / A_{s}$ is given by

$$
\rho=\frac{t_{s}}{t_{p}} \frac{\frac{\cos ^{3} \theta_{i}}{\cos \theta_{t}} L_{X X}^{2} \operatorname{Im}\left[\chi_{X X}^{(1)}\right]+\frac{n_{a}}{n_{b}} \sin ^{2} \theta_{i} L_{Z Z}^{2} \operatorname{Im}\left[\chi_{Z Z}^{(1)}\right]}{L_{Y Y}^{2} \operatorname{Im}\left[\chi_{Y Y}^{(1)}\right]}
$$

To understand the meaning of this equation, we define the effective incident angle $\chi^{\prime}$ and the $p / s$ enhancement factor $a_{p / s}$ as

$$
\begin{aligned}
& \chi^{\prime}=\tan ^{-1}\left[\sqrt{\frac{n_{a}}{n_{b}} \sin ^{2} \theta_{i} L_{Z Z}^{2} / \frac{\cos ^{3} \theta_{i}}{\cos \theta_{t}} L_{X X}^{2}}\right] \\
& a_{p / s}=\frac{t_{s}}{t_{p}}\left(\frac{\cos ^{3} \theta_{i}}{\cos \theta_{t}} L_{X X}^{2}+\frac{n_{a}}{n_{b}} \sin ^{2} \theta_{i} L_{Z Z}^{2}\right) \frac{1}{L_{Y Y}^{2}}
\end{aligned}
$$


Then, Eq. (S.9) can be re-written as

$$
\rho=a_{p / s} \frac{\cos ^{2} \chi^{\prime} \cdot \operatorname{Im}\left[\chi_{X X}^{(1)}\right]+\sin ^{2} \chi^{\prime} \cdot \operatorname{Im}\left[\chi_{Z Z}^{(1)}\right]}{\operatorname{Im}\left[\chi_{Y Y}^{(1)}\right]}
$$

Assuming that the microscopic local field correction factor is isotropic as justified later, using the dependence of $\ddot{\chi}^{(1)}$ on the average molecular orientation, ${ }^{3}$ this can be further written as

$$
\rho=a_{p / s} \frac{\cos ^{2} \chi^{\prime} \cdot \frac{1}{2}\left\langle\sin ^{2} \theta\right\rangle+\sin ^{2} \chi^{\prime} \cdot\left\langle\cos ^{2} \theta\right\rangle}{\frac{1}{2}\left\langle\sin ^{2} \theta\right\rangle}=a_{p / s}\left(1+3 \sin ^{2} \chi^{\prime} \cdot \frac{\langle S\rangle}{1-\langle S\rangle}\right)
$$

where $\theta$ is an angle between a transition dipole moment and the surface normal, and $\langle\ldots\rangle$ takes the ensemble average. $\langle S\rangle \equiv\left\langle\left(3 \cos ^{2} \theta-1\right) / 2\right\rangle$ is the order parameter, which is determined based on the linear dichroism measurement and used to extract correlation functions from PSAR-PP experiment.

It is instructive to compare (S.11) with a special case of a polarization sheet embedded in two completely index matched materials, i.e. $n_{a}=n_{b}=n^{\prime}$. In this case, there is no refraction and reflection of the beams, and the dichroic ratio is given by

$$
\rho_{\mathrm{im}}=\frac{\cos ^{2} \chi \cdot \operatorname{Im}\left[\chi_{X X}^{(1)}\right]+\sin ^{2} \chi \cdot \operatorname{Im}\left[\chi_{\mathrm{ZZ}}^{(1)}\right]}{\operatorname{Im}\left[\chi_{Y Y}^{(1)}\right]}
$$

where $\chi=\theta_{i}$ is the incident angle. Comparing (S.11) and (S.13), we can deduce for the general case that in the context of comparing $p$ - and $s$-polarization absorption, (1) the field is effectively oriented in the layer as if the beam is propagating in the layer with the effective incident angle $\chi^{\prime}$, instead of the actual incident angle $\chi$ in the air, and (2) the local field enhancement adds up to enhance the $p$-polarization absorption by the factor of $a_{p / s}$ relative to the $s$-polarization absorption. This intuitive interpretation applies for the local field effect in PSAR-PP experiments as well.

We would like to note that the effective incident angle $\chi^{\prime}$ and the $p / s$ enhancement factor $a_{p / s}$ can be equivalently written as

$$
\begin{aligned}
& \chi^{\prime}=\tan ^{-1}\left[\sqrt{\sin \theta_{i} \sin \theta_{t} L_{Z Z}^{(1)} L_{Z Z}^{(2)} / \cos \theta_{i} \cos \theta_{t} L_{X X}^{(1)} L_{X X}^{(2)}}\right] \\
& a_{p / s}=\frac{t_{s}}{t_{p}}\left(\sin \theta_{i} \sin \theta_{t} L_{Z Z}^{(1)} L_{Z Z}^{(2)}+\cos \theta_{i} \cos \theta_{t} L_{X X}^{(1)} L_{X X}^{(2)}\right) \frac{1}{L_{Y Y}^{(1)} L_{Y Y}^{(2)}},
\end{aligned}
$$


where $\vec{L}^{(1)}$ is the local field correction factor for the polarization induction process which is identical to Eqs. (S.3a)-(S.3c), while $\vec{L}^{(2)}$ is the local field correction factor for the field emission process from the polarization sheet to the substrate, which is obtained by exchanging $n_{a} \leftrightarrow n_{b}$ and $\theta_{i} \leftrightarrow \theta_{t}$ in Eqs. (S.3a)-(S.3c). This notation may appear more intuitive to the readers familiar with Shen and Heinz's work. ${ }^{1,2}$

\section{A2. Effective Refractive Index and Microscopic Local Field}

To calculate $L_{Z Z}$ in Eq. (S.3c), it is necessary to specify the effective refractive index of the thin layer $n^{\prime}$. Zhuang et al. suggests that this refractive index can be chosen such that ${ }^{3,4}$

$$
n^{\prime}=\sqrt{\frac{n_{a}{ }^{2}+n_{b}{ }^{2}+4}{2\left(n_{a}{ }^{-2}+n_{b}{ }^{-2}+1\right)}}
$$

This refractive index, under modified Lorentz model, makes the microscopic local field correction factors isotropic. Therefore, with the adoption of Eq. (S.15), the microscopic local field correction can be neglected when a polarization dependence of a signal intensity is studied. For our system in Figure S1, Eq. (S.15) yields $n^{\prime}=1.178$. We decided to adopt this value of the effective refractive index for our analysis for the following reasons.

(1) Zhuang et al. studied a hexadecanol monolayer at water-air interface, and compared the orientation of molecules determined from SFG spectroscopy and X-ray diffraction measurement. ${ }^{4}$ The orientation of the terminal $-\mathrm{CH}_{3}$ group located right at the monolayer-air interface was consistent with the X-ray diffraction measurement within the error when the effective refractive index was chosen as in Eq. (S.15). They also indicated that the value of the effective refractive index appears to be independent of the vibrational probes' chemical structures and the locations within the interface. ${ }^{4}$

(2) Most importantly, when the effective refractive index $n^{\prime}$ was chosen to be this value, the correlation functions in PSAR-PP spectroscopy acquired from two different incident angles measurements $\left(60^{\circ}\right.$ and $\left.45^{\circ}\right)$ yielded consistent results (Figure S6).

\section{A3. Calculated Effective Incident Angle and $p / s$ Enhancement Factor}

With the use of $n^{\prime}=1.178$, Table S1 shows the effective incident angle $\chi^{\prime}$ and the $p / s$ enhancement factor $a_{p / s}$ in Eqs. (S.10) for various incident angles $\chi$. These values were used for the following analysis of the IRLD and PSAR-PP results. It is worth noting that while the effective incident angle $\chi^{\prime}$ is significantly different from $\chi$ as a consequence of $n^{\prime}>1, p / s$ enhancement factor $a_{p / s}$ is close to unity even at relatively large incident angle of $60^{\circ}$. 


\begin{tabular}{|c|c|c|}
\hline $\begin{array}{l}\text { Incident Angle } \\
\text { in Air } \chi\end{array}$ & $\begin{array}{l}\text { Effective Incident } \\
\text { Angle } \chi^{\prime}\end{array}$ & $\begin{array}{l}p / s \text { enhancement } \\
\text { factor } a_{p / s}\end{array}$ \\
\hline $0^{\circ}$ & $0^{\circ}$ & 1 \\
\hline $10^{\circ}$ & $8.529^{\circ}$ & 1.00 \\
\hline $20^{\circ}$ & $17.01^{\circ}$ & 1.002 \\
\hline $30^{\circ}$ & $25.39^{\circ}$ & 1.004 \\
\hline $40^{\circ}$ & $33.63^{\circ}$ & 1.006 \\
\hline $45^{\circ}$ & $37.70^{\circ}$ & 1.007 \\
\hline $50^{\circ}$ & $41.73^{\circ}$ & 1.009 \\
\hline $60^{\circ}$ & $49.72^{\circ}$ & 1.011 \\
\hline
\end{tabular}

Table S1. The effective incident angle $\chi^{\prime}$ and the $p / s$ enhancement factor $a_{p / s}$ in Eqs. (S.10) calculated for various incident angles in the air $\chi . n^{\prime}=1.178$ was used as discussed in Sec. A2.

\section{A4. Macroscopic Local Field Effect for PSAR-PP Spectroscopy}

The local field correction scheme discussed above applies to PSAR-PP spectroscopy as well. Our goal is to derive Eqs. (S.21a) and (S.21b). Figure S2 shows the beam and the polarization scheme used for "parallel" and "perpendicular" signals, respectively. We will obtain the local field correction factors for the general probe incident angle $\theta_{i}$. As discussed in the main text, the pump pulse is always set to be $s$-polarized. The probe pulse is $s$-polarized when the parallel signal is recorded (Fig. S2, left), and is $p$-polarized when the perpendicular signal is recorded (Fig. S2, right).

In the case of PSAR-PP spectroscopy, what is detected is the signal field emitted toward the substrate from the interfacial third-order polarization $\vec{P}^{(3)^{\prime}}$ induced by two interactions from the pump pulse and one interaction from the probe pulse, both incident from the air. Again, the emitted signal collinearly propagates with the transmitted probe field.

The overall transmitted field including the emission from the third-order polarization $\vec{P}^{(3)^{\prime}}$ can be readily obtained by simply replacing $\vec{P}^{(1)^{\prime}}$ in Eq. (S.1) with $\vec{P}^{(3)^{\prime}}$ :

$$
\begin{aligned}
E_{t, \mathrm{para}} & =t_{s} E_{\mathrm{pr}, s}+E_{\mathrm{para}}^{(3)} \\
& =t_{s} E_{\mathrm{pr}, s}+\frac{i \omega}{2 c \varepsilon_{0} n_{a} \cos \theta_{i}} L_{Y Y} P_{Y}^{(3)^{\prime}}
\end{aligned}
$$




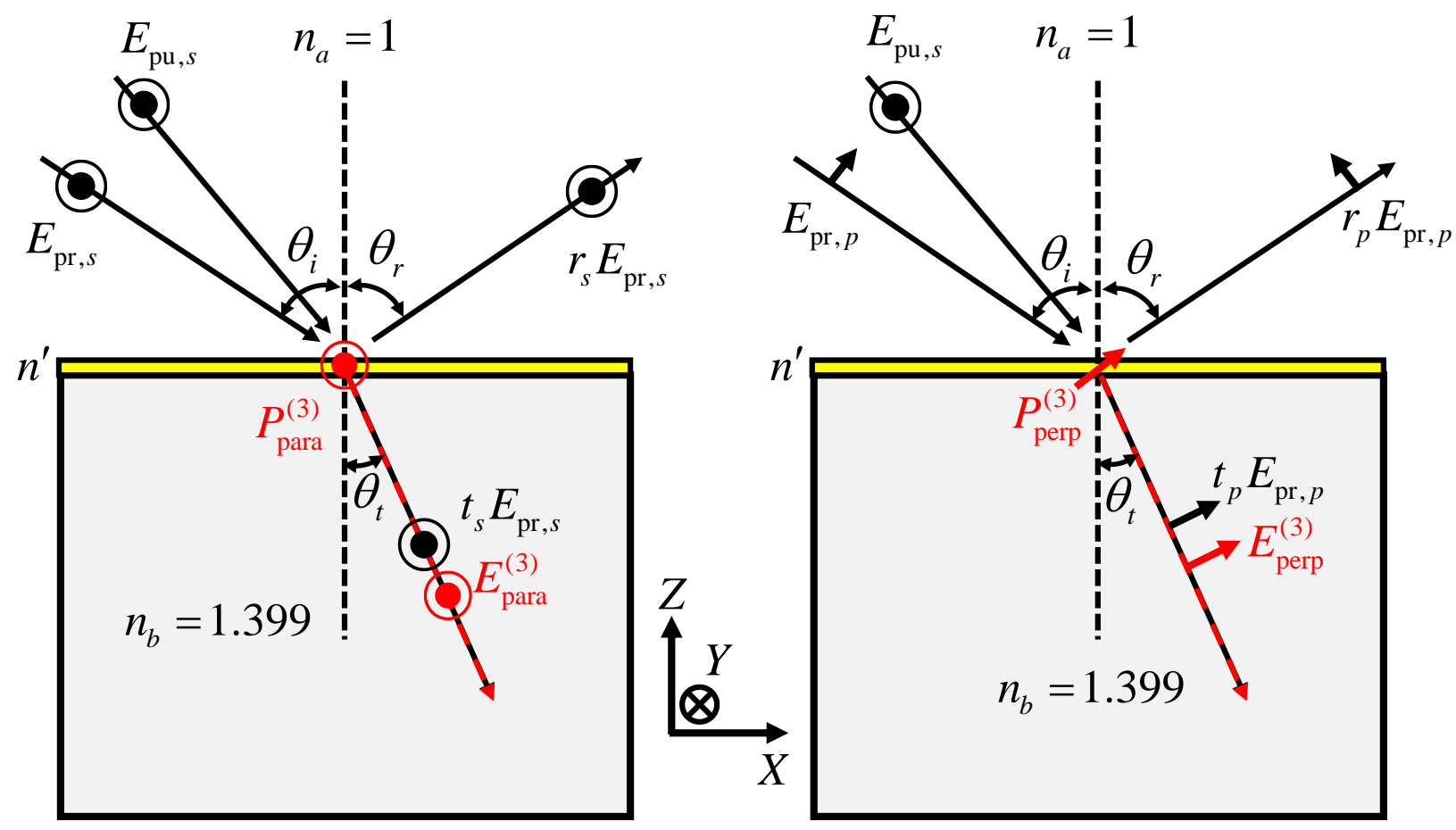

Figure S2. PSAR-PP experiment on an interfacial layer for the measurement of the "parallel" signal (left) and the "perpendicular" signal (right). The $s$-polarized pump beam and either $s$-polarized (parallel) or $p$ polarized (perpendicular) probe beam induces the third-order polarization $P^{(3)}$, which emits the signal field $E^{(3)}$. The $E^{(3)}$ interferes with the transmitted probe field to be heterodyned-detected. The angles written in these figures are those for the probe beam, which are the only relevant angles for the data analysis. The reflected/transmitted pump beams are omitted for clarity.

$$
\begin{aligned}
E_{t, \text { perp }} & =t_{p} E_{\mathrm{pr}, p}+E_{\mathrm{perp}}^{(3)} \\
& =t_{p} E_{\mathrm{pr}, p}+\frac{i \omega}{2 c \varepsilon_{0} n_{a} \cos \theta_{i}}\left[\frac{\cos \theta_{i}}{\cos \theta_{t}} L_{X X} P_{X}^{(3)^{\prime}} \cos \theta_{i}+\frac{n_{a}}{n_{b}} L_{Z Z} P_{Z}^{(3)^{\prime}} \sin \theta_{i}\right]
\end{aligned}
$$

where $t_{s}, t_{p}$ and $\vec{L}$ are identical to those in Eqs. (S.2) and (S.3) with $\theta_{i}, \theta_{t}$ and $\theta_{r}$ being for the probe beam. $E_{\mathrm{pr}, s}$ and $E_{\mathrm{pr}, p}$ are the amplitudes of the $s$-polarized (for "parallel" measurement) and the $p$-polarized (for "perpendicular" measurement) probe incident field in the air.

The interfacial third-order polarization $\vec{P}^{(3)^{\prime}}$ is given as

$$
\begin{aligned}
& P_{X}^{(3)^{\prime}}=\varepsilon_{0} \chi_{X X Y Y}^{(3)}\left(L_{X X} \cos \theta_{i} E_{\mathrm{pr}, p}\right)\left(L_{Y Y}{ }^{\prime} E_{\mathrm{pu}, s}\right)\left(L_{Y Y}{ }^{\prime} E_{\mathrm{pu}, s}\right) \\
& P_{Y}^{(3)^{\prime}}=\varepsilon_{0} \chi_{Y Y Y Y}^{(3)}\left(L_{Y Y} E_{\mathrm{pr}, s}\right)\left(L_{Y Y}{ }^{\prime} E_{\mathrm{pu}, s}\right)\left(L_{Y Y}{ }^{\prime} E_{\mathrm{pu}, s}\right)
\end{aligned}
$$




$$
P_{Z}^{(3)^{\prime}}=\varepsilon_{0} \chi_{Z Z Y Y}^{(3)}\left(L_{Z Z} \sin \theta_{i} E_{\mathrm{pr}, p}\right)\left(L_{Y Y}{ }^{\prime} E_{\mathrm{pu}, s}\right)\left(L_{Y Y}{ }^{\prime} E_{\mathrm{pu}, s}\right)
$$

$\vec{L}^{\prime}$ is the local field correction factor for the pump pulse, which can be obtained from Eq. (S.3) by replacing $\left(\theta_{i}, \theta_{t}\right)$ with $\left(\theta_{i}^{\prime}, \theta_{t}^{\prime}\right)$, namely the incident and the transmission angle for the pump beam. Only $y$-component $L_{Y Y}{ }^{\prime}$ is of concern here because the pump beam is always $s$-polarized. As shown later, this $L_{Y Y}{ }^{\prime}$ will be combined with the geometry factors, which are experimentally accounted for in the scaling process, and thus do not have to be calculated.

As discussed in the main text, the pump pulse was turned on/off every other shot and the pumpprobe signal was recorded as $\left(I_{\text {on }}-I_{\text {off }}\right) / I_{\text {off }}$. Therefore, the "parallel" signal $R_{\|}$is given by

$$
\begin{aligned}
R_{\|} & =\frac{\left|t_{s} E_{\mathrm{pr}, s}+E_{\mathrm{para}}^{(3)}\right|^{2}-\left|t_{s} E_{\mathrm{pr}, s}\right|^{2}}{\left|t_{s} E_{\mathrm{pr}, s}\right|^{2}} \approx 2 \operatorname{Re}\left[t_{s} E_{\mathrm{pr}, s} E_{\mathrm{para}}^{(3)} /\left|t_{s} E_{\mathrm{pr}, s}\right|^{2}\right] \\
& =\frac{2}{t_{s}} \cdot \frac{i \omega}{2 c n_{a} \cos \theta_{i}} \chi_{Y Y Y Y}^{(3)} L_{Y Y}^{2}\left(L_{Y Y}{ }^{\prime} E_{\mathrm{pu}, s}\right)^{2}
\end{aligned}
$$

The same calculation applies for the "perpendicular" signal $R_{\perp}$ to yield

$$
R_{\perp}=\frac{2}{t_{p}} \cdot \frac{i \omega}{2 c n_{a} \cos \theta_{i}}\left[\frac{\cos ^{3} \theta_{i}}{\cos \theta_{t}} L_{X X}^{2} \chi_{X X Y Y}^{(3)}+\frac{n_{a}}{n_{b}} \sin ^{2} \theta_{i} L_{Z Z}^{2} \chi_{\mathrm{ZZYY}}^{(3)}\right]\left(L_{Y Y}{ }^{\prime} E_{\mathrm{pu}, s}\right)^{2}
$$

By defining the geometry factor $A\left(\theta_{i}, \theta_{i}^{\prime}, E_{\mathrm{pu}, s}\right)$ as

$$
A\left(\theta_{i}, \theta_{i}^{\prime}, E_{\mathrm{pu}, s}\right) \equiv \frac{2}{t_{s}} \cdot \frac{i \omega}{2 c n_{a} \cos \theta_{i}} L_{Y Y}^{2}\left(L_{Y Y}{ }^{\prime} E_{\mathrm{pu}, s}\right)^{2}
$$

$R_{\|}$and $R_{\perp}$ can be written as

$$
\begin{aligned}
& R_{\|}=A\left(\theta_{i}, \theta_{i}^{\prime}, E_{\mathrm{pu}, s}\right) \operatorname{Im}\left[\chi_{Y Y Y Y}^{(3)}\right] \\
& R_{\perp}=A\left(\theta_{i}, \theta_{i}^{\prime}, E_{\mathrm{pu}, s}\right) a_{p / s}\left[\cos ^{2} \chi^{\prime} \cdot \operatorname{Im}\left[\chi_{X X Y Y}^{(3)}\right]+\sin ^{2} \chi^{\prime} \cdot \operatorname{Im}\left[\chi_{Z Z Y Y}^{(3)}\right]\right]
\end{aligned}
$$

Again, Eq. (S.21) can be viewed as if the effective incident angle in the polarization sheet is altered from $\chi$ to $\chi^{\prime}$, and the signal for the $p$-polarized probe beam is enhanced by $a_{p / s}$ relative to the $s$-polarized probe beam. The third-order susceptibility in Eq. (S.21) is exactly what was evaluated in a previous publication, ${ }^{6}$ and these signals can be more explicitly written using the order parameter $\langle S\rangle$, the out-of-plane correction function $C^{\text {op }}(t)$ (Eq. (4.1)), the mainly-in-plane correlation function $C^{\text {mip }}(t)$ (Eq. (4.2)), and the population decay $P(t)$ as 


$$
\begin{aligned}
R_{\|}(t)=A\left(\theta_{i}, \theta_{i}^{\prime}, E_{\mathrm{pu}, s}\right) \times\left[\frac{1}{9}-\frac{2}{9}\langle S\rangle+\frac{1}{9} C^{\mathrm{op}}(t)+\frac{1}{8} C^{\mathrm{mip}}(t)\right] \times P(t) \\
R_{\perp}(t)=A\left(\theta_{i}, \theta_{i}^{\prime}, E_{\mathrm{pu}, s}\right) \times a_{p / s} \times \\
\quad\left[\frac{1}{9}-\frac{1}{9}\left(3 \cos ^{2} \chi^{\prime}-1\right)\langle S\rangle+\frac{1}{9}\left(3 \cos ^{2} \chi^{\prime}-2\right) C^{\mathrm{op}}(t)-\frac{1}{8}\left(\cos ^{2} \chi^{\prime}\right) C^{\mathrm{mip}}(t)\right] \times P(t)
\end{aligned}
$$

As discussed in the main text, PSAR-PP spectroscopy is implemented in two geometries, namely the "normal" (Fig. 3A) and the "tilted" (Fig. 3B) geometries. For the normal geometry, $\chi^{\prime}=\chi=0^{\circ}$ and $a_{p / s}=1$, which yields Eqs. (3.1) and (3.2) in the main text where the geometry factor is written simply as $A$. There is no $p / s$ enhancement factor involved for the normal geometry because all the polarizations involved are $s$-polarized. The geometry factor for the tilted geometry is simply written as $B$, which yields Eqs. (3.3) and (3.4). 


\section{B. Sample Preparation}

The complete details of the sample preparation procedure can be found in our previous publications. ${ }^{7}$ Here, a brief summary of the synthesis procedure is provided. In the following procedure, unless specifically mentioned, the chemicals were obtained from Sigma Aldrich and used without further purifications.

A CaF 2 wafer (1" diameter, 1 wavelength flatness at $633 \mathrm{~nm}$, NewRise) was coated with a 100 $\mathrm{nm} \mathrm{SiO} 2$ layer by plasma-enhanced chemical vapor deposition in UCSB Nanofabrication Facility. $10 \mu \mathrm{L}$ of 11-bromoundecyltrichlorosilane (Br- $\mathrm{C}_{11}-\mathrm{SiCl}_{3}$, Gelest) was added to $10 \mathrm{~mL}$ of bicyclohexyl, and the $\mathrm{SiO}_{2} / \mathrm{CaF}_{2}$ wafer was immersed in this solution for 1 hour. The selfassembled $\mathrm{Br}-\mathrm{C}_{11}$ monolayer on the $\mathrm{SiO}_{2} / \mathrm{CaF}_{2}$ wafer was then immersed in a $20 \mathrm{~mL}$ of saturated dimethylformamide (DMF) solution of sodium azide for 24 hours to replace the terminal bromine with an azide $\left(-\mathrm{N}_{3}\right)$ group through an $\mathrm{S}_{\mathrm{N}} 2$ reaction. The terminal azide on the monolayer was then used for a copper-catalyzed azide-alkyne cycloaddition (CuAAC) "click" reaction with fac- $\mathrm{Re}(\mathrm{phenC} \equiv \mathrm{CH})(\mathrm{CO})_{3} \mathrm{Cl}$, which was prepared as in a previous study, ${ }^{8}$ to yield the monolayer in Fig. 1(a) shown in the main text. The synthesized monolayer was immersed in chloroform for 1 hour to remove unclicked metal carbonyl complexes from the surface. It has been shown by AFM imaging that the surface of the sample prepared in this manner is very flat, namely $0.42 \mathrm{~nm}$ rms variation over a $10 \mathrm{~nm}$ correlation length, which corresponds to only $2^{\circ}$ variation of the surface. ${ }^{9}$

As discussed in Section F, to estimate the contribution from Förster excitation transfer, the sample with the reduced head group density was prepared as well. This sample was prepared by replacing the 11-bromoundecyltrichlorosilane/bicyclohexyl solution above with the mixture of 5 $\mu \mathrm{L}$ of 11-bromoundecyltrichlorosilane $\left(\mathrm{Br}^{-} \mathrm{C}_{11}-\mathrm{SiCl}_{3}\right)$ and $5 \mu \mathrm{L}$ of undecyltrichlorosilane $\left(\mathrm{C}_{11^{-}}\right.$ $\mathrm{SiCl}_{3}$, Gelest) in $10 \mathrm{~mL}$ of bicyclohexyl. All the other procedures are identical. FT-IR spectrum verified that the head group density for this sample is $\sim 70 \%$ of the density in the sample with the full functionalization. 


\section{Time-averaged Infrared Linear Dichroism}

\section{C1. Experimental}

Nicolet 6700 FT-IR spectrometer (Thermo Scientific) was used to measure infrared absorption spectra with $2 \mathrm{~cm}^{-1}$ resolution and 1024 scans. A $\mathrm{CaF}_{2}$ holographic wire grid polarizer (Thorlabs) was installed in the beam path before the beam passes through the sample. The samples were installed on a rotation mount to control the incident angle of the beam.

The measurement of the absorption spectra such as in Fig. 1(b) requires the measurement of the background intensity $\left(I_{\mathrm{bg}}\right)$ and the sample intensity $\left(I_{\text {smpl }}\right)$. The background intensity $I_{\mathrm{bg}}$ was obtained as the IR beam intensity passing through a $\mathrm{SiO}_{2} / \mathrm{CaF}_{2}$ wafer without the monolayer grafted on to it, and the sample intensity $I_{\text {smpl }}$ was acquired as the intensity passing through the $\mathrm{SiO}_{2} / \mathrm{CaF}_{2}$ wafer with the functionalized monolayer. The absorbance was then obtained as $A=-\log _{10}\left(I_{\mathrm{smpl}} / I_{\mathrm{bg}}\right)$.

The absorbance was measured for the $s$-polarized and $p$-polarized incident beam with various incident angles from $0^{\circ}$ to $60^{\circ}$ with $10^{\circ}$ increments. The measured absorbance for each polarization was integrated from $2018.5 \mathrm{~cm}^{-1}$ to $2028.5 \mathrm{~cm}^{-1}\left(2023.5 \pm 5 \mathrm{~cm}^{-1}\right)$, yielding $A_{s}$ for the $s$-polarized beam and $A_{p}$ for the $p$-polarized beam. For each incident angle, the dichroic ratio $\rho=A_{p} / A_{s}$ was calculated. The result is plotted in Figure S3(a).

\section{C2. Incident Angle Dependence of Dichroic Ratio and Order Parameter}

As shown in Section A1, the measured dichroic ratio is related to the order parameter $\langle S\rangle$ by Eq. (S.12). By simple manipulation of Eq. (S.12), we obtain

$$
\frac{\rho}{a_{p / s}}=1+3 \sin ^{2} \chi^{\prime} \cdot \frac{\langle S\rangle}{1-\langle S\rangle}
$$

For each incident angle in the air $\chi$, the effective incident angle $\chi^{\prime}$ and the $p / s$ enhancement factor $a_{p / s}$ can be obtained as calculated in Table S1, with the effective refractive index of $n^{\prime}=1.178$. In Figure S3(b), the left hand side in Eq. (S.23) with the measured dichroic ratio is plotted with respect to the effective incident angle. The red line is the best fit to the data using the right hand side of Eq. (S.23), which yields the order parameter $\langle S\rangle=-0.290 \pm 0.016$. As discussed in the main text, this order parameter was used for the analysis of PSAR-PP experiment. 

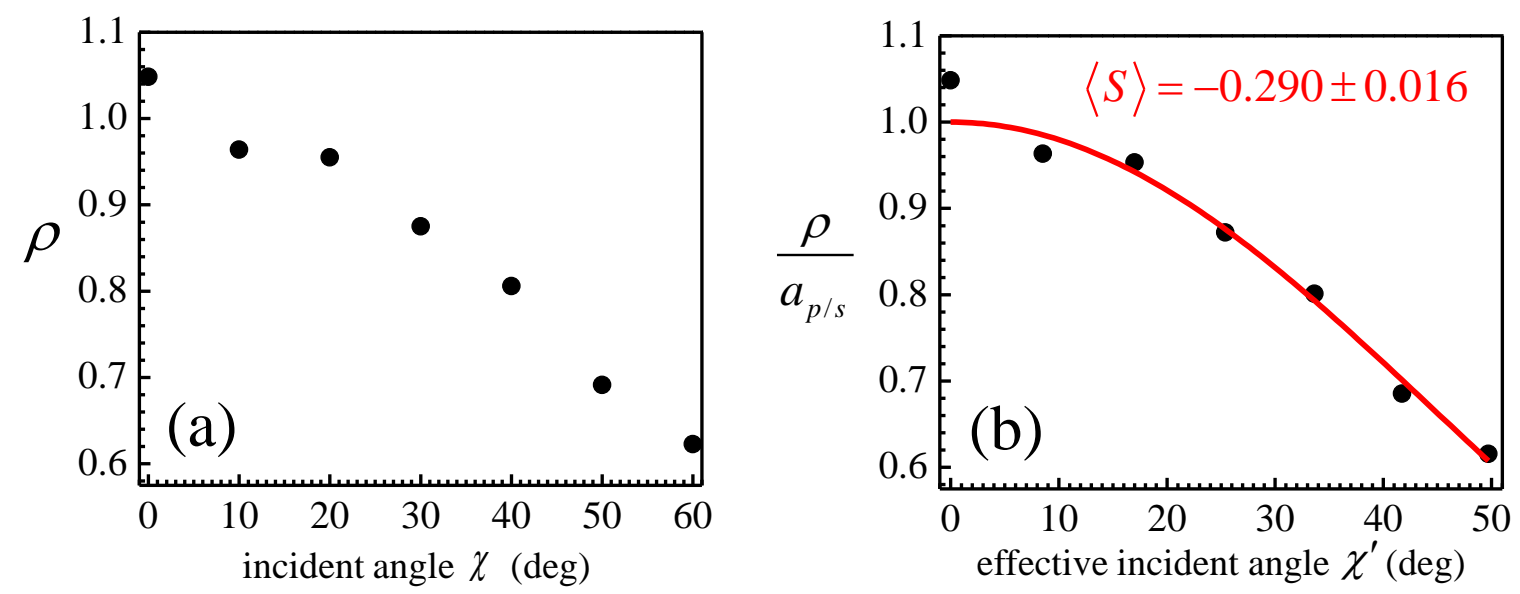

Figure S3. (a) The measured dichroic ratio $A_{p} / A_{s}$ for various incident angles in the air $\chi$. (b) Dots - the measured dichroic ratio in Fig. S3(a) divided by $p / s$ enhancement factor $a_{p / s}$ with respect to the effective incident angle $\chi^{\prime}$. Note that $a_{p / s}$ and $\chi^{\prime}$ depend on the incident angle and tabulated in Table S1. Red line - the fit of the observed $\rho / a_{p / s}$ to the right hand side of Eq. (S.23). The best fit was obtained with $\langle S\rangle=-0.290$, and the $1 \sigma$ fitting error was estimated to be 0.016 . 


\section{Polarization-selective Angle-resolved Pump-probe (PSAR-PP) Spectroscopy}

\section{D1. Experimental}

A regenerative amplifier system (Spitfire Ace, Spectra Physics), seeded by a $83 \mathrm{MHz}$ oscillator output (MaiTai, Spectra Physics) and pumped by a 25W Nd:YLF Q-switch laser output (Empower, Spectra Physics) yielded a $800 \mathrm{~nm}$ pulse with the time duration of sub-70 fs, the pulse energy of $2 \mathrm{~mJ}$, and the repetition rate of $3 \mathrm{kHz}$. This amplified $800 \mathrm{~nm}$ pulse was used to pump a home built optical-parametric amplifier (OPA) based on $3 \mathrm{~mm}$-thick BBO crystals to generate a near-infrared signal pulse and an idler pulse with the total energy of $500 \mu \mathrm{J}$. The signal and idler pulses were then spatially and temporarily overlapped and collinearly sent to a $1.5 \mathrm{~mm}$-thick $\mathrm{AgGaS}_{2}$ crystal to yield a mid-infrared pulse $(\sim 33 \mu \mathrm{J}, \sim 170 \mathrm{fs})$ centered at 2030 $\mathrm{cm}^{-1}$ with the spectral bandwidth of $90 \mathrm{~cm}^{-1}$ fwhm.

The generated pulse was split into a stronger pump pulse and a weaker probe pulse by 92:8 ZnSe beam splitter. The pump pulse was sent to a pulse shaper system based on a germanium acoustooptic modulator (LS700-1109, Isomet). The pulse shaper system is essentially identical to the one described in a previous publication, ${ }^{10}$ except that as an arbitrary waveform generator we employed PXDAC4800 from Signatec. Using the pulse shaper system, we modified the spectrum of the excitation pulse such that $<1950 \mathrm{~cm}^{-1}$ component of the spectrum was eliminated. Thus, the low frequency modes of the metal complex are not pumped by the pump pulse, which potentially interferes with the signal of interest through coherent transfer and population transfer processes. ${ }^{11-13}$ The pulse shaper generates the pump pulse every other shot, and the phase of the pump pulse was flipped by $\pi$ for every other generated pump pulse. Overall, the output from the pulse shaper system is a 4-shot sequence represented as ( $0, \mathrm{OFF}, \pi, \mathrm{OFF})$, where 0 and $\pi$ are the phase of the pump pulse and "OFF" stands for the pump pulse not being generated. This sequence is useful to remove minor scattered light from the surface, which interferes with the pump-probe signal. ${ }^{12}$

The probe pulse is delayed by a controllable linear delay line. The pump pulse as the output from the pulse shaper system, and the probe pulse crossed in the sample as shown in Figure 2 in the main text. The cross correlation between the pump pulse and the probe pulse yielded the effective pulse duration of $\sim 190$ fs. To make the pump pulse $s$-polarized, a $\mathrm{MgF}_{2}$ half-wave plate was installed in the pump path. The polarizers used in Figure 2 are all high-contrast wire grid silicon polarizers (POL-3-5-SI-25, ISP optics). The resolving polarizer is mounted on a motorized polarizer rotation mount, and switched during the measurement to either $s$-polarized or $p$-polarized to record parallel or perpendicular signals, respectively. The probe passing through the resolving polarizer was projected to $45^{\circ}$ by another polarizer and sent to a spectrograph to disperse the frequency component of the probe pulse, and detected by a 32element $\mathrm{HgCdTe}$ array detector. In the following and in the main text, we are discussing the pump-probe signal at the probe frequency of $2023.5 \mathrm{~cm}^{-1}$. The pump-probe signal was recorded as in Eq. (2) in the main text.

To account for a shot-to-shot instability of the mid-infrared pulses, a $\mathrm{CaF}_{2}$ window was installed in the probe path with $45^{\circ}$ incident angle to reflect a minor fraction of the probe pulse, and the 
reflection was monitored by a single element $\mathrm{HgCdTe}$ detector as a reference detector. The sensitivity was significantly improved by this referencing scheme to detect a minute signal arising from a single layer of molecules.

\section{D2. Saturation Effect}

As briefly mentioned in the main text, care needs to be paid to avoid "saturation" of the IR transition. Pump-probe spectroscopy essentially probes the number of molecules in the excited state. When a significant portion of the molecules in the beam focus is pumped into the excited state, the number of molecules in the excited state is no longer proportional to $\left(\vec{\mu} \cdot \vec{E}_{\text {pump }}\right)^{2}$. The proportionality between the signal and $\left(\vec{\mu} \cdot \vec{E}_{\text {pump }}\right)^{2}$ is a fundamental assumption made to derive the polarization dependence of the pump-probe signals, such as Eq. (3) in the main text for interfacial molecules, or the anisotropy decay for isotropic samples given by

$$
r(t) \equiv \frac{R_{\|}-R_{\perp}}{R_{\|}+2 R_{\perp}}=0.4 C_{2}(t)
$$

Thus, under strong saturation, these schemes to extract orientational correlation functions are no longer valid. This saturation effect emerges as a pump intensity dependence of the observed anisotropy decays. In "parallel" signals, the probe tends to read out the molecules pumped with large $\left(\vec{\mu} \cdot \vec{E}_{\text {pump }}\right)^{2}$, while in "perpendicular" signals, the probe reads out the molecules pumped with smaller $\left(\vec{\mu} \cdot \vec{E}_{\text {pump }}\right)^{2}$, simply because the pump and the probe polarizations are orthogonal. As a result, the saturation effect reduces the amplitude of the parallel signal more than the perpendicular signal, and therefore the observed anisotropy emerges lower than $0.4 C_{2}(t)$.

It is thus important to keep the pump power low enough not to saturate the transition of interest. To quantitatively evaluate the extent of the saturation with a good signal-to-noise ratio, we prepared a solution of $\mathrm{Re}(\mathrm{phenC} \equiv \mathrm{CH})(\mathrm{CO})_{3} \mathrm{Cl}$ (used for the synthesis of the monolayer) in dimethyl sulfoxide (DMSO) and applied a standard polarization-selective pump-probe (PSPP) spectroscopy to the CO symmetric stretching mode. Because the solution is isotropic, the anisotropy in Eq. (S.24) should be evaluated to obtain the orientational correlation function. The anisotropies of this bulk solution measured for various pump intensities are plotted in Figure S4. Note that the intensities shown in the legend of the plot are rough estimates, while the ratios between the intensities are accurate. Clearly, the observed anisotropy strongly depends on the pump intensity, and becomes lower as the pump intensity increases, which is qualitatively 


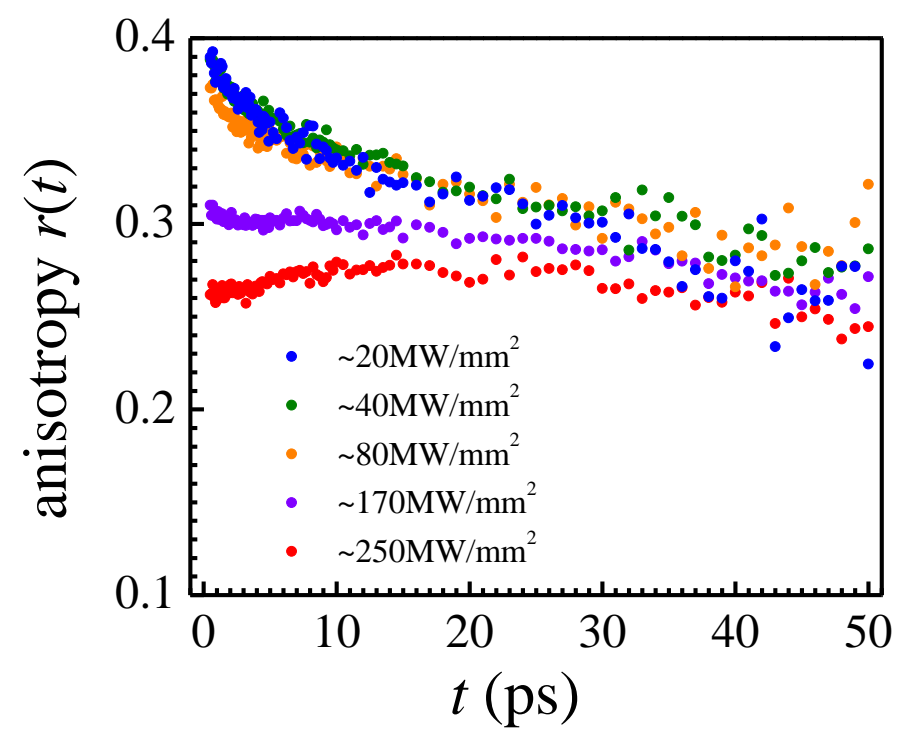

Figure S4. The pump intensity dependence of the observed anisotropy for the bulk solution of $\mathrm{Re}(\mathrm{phenC} \equiv \mathrm{CH})(\mathrm{CO})_{3} \mathrm{Cl}$ in DMSO. Note that the intensities in the legend are very rough estimates and may not be absolutely accurate. As expected from the saturation effect, the higher pump intensity significantly reduces the anisotropy value. At $\sim 40 \mathrm{MW} / \mathrm{mm}^{2}$ the transition dipole is free from the saturation effect. The same pump intensity $\sim 40 \mathrm{MW} / \mathrm{mm}^{2}$ was employed for PSAR-PP experiments on the monolayer sample.

explained by the saturation effect. So far there is no analytical model established to handle the observed anisotropy decay under the strong saturation effect. As seen in Figure S4, 20 $\mathrm{MW} / \mathrm{mm}^{2}$ pump and $\sim 40 \mathrm{MW} / \mathrm{mm}^{2}$ pump essentially yielded the identical decay, indicating that the transition is free from the saturation effect once the pump intensity is reduced to $\sim 40$ $\mathrm{MW} / \mathrm{mm}^{2}$. Also, these two anisotropy decays extrapolate to 0.4 at $t=0$, which is the maximum possible value of the anisotropy for any isotropic samples, further verifying that these signals are completely free from the saturation effect. In the actual PSAR-PP experiment for the monolayer, the pump intensity identical to $\sim 40 \mathrm{MW} / \mathrm{mm}^{2}$ was employed to make sure the acquired signal is free from saturation.

Note that DMSO has a relatively high refractive index of $\sim 1.5$, which is significantly higher than the effective refractive index at the monolayer-air interface (1.178). Therefore the microscopic local field experienced by the metal carbonyl vibrational probe is expected to be lower at monolayer-air interface than in DMSO.

\section{D3. Tilt Angle Dependence}

As discussed in depth in the main text, the out-of-plane correlation function $C^{\mathrm{op}}(t)$ and the mainly-in-plane correlation function $C^{\text {mip }}(t)$ were extracted by solving the simultaneous linear equations in Eqs. (3) with the experimental observables. To achieve this, the parallel and 

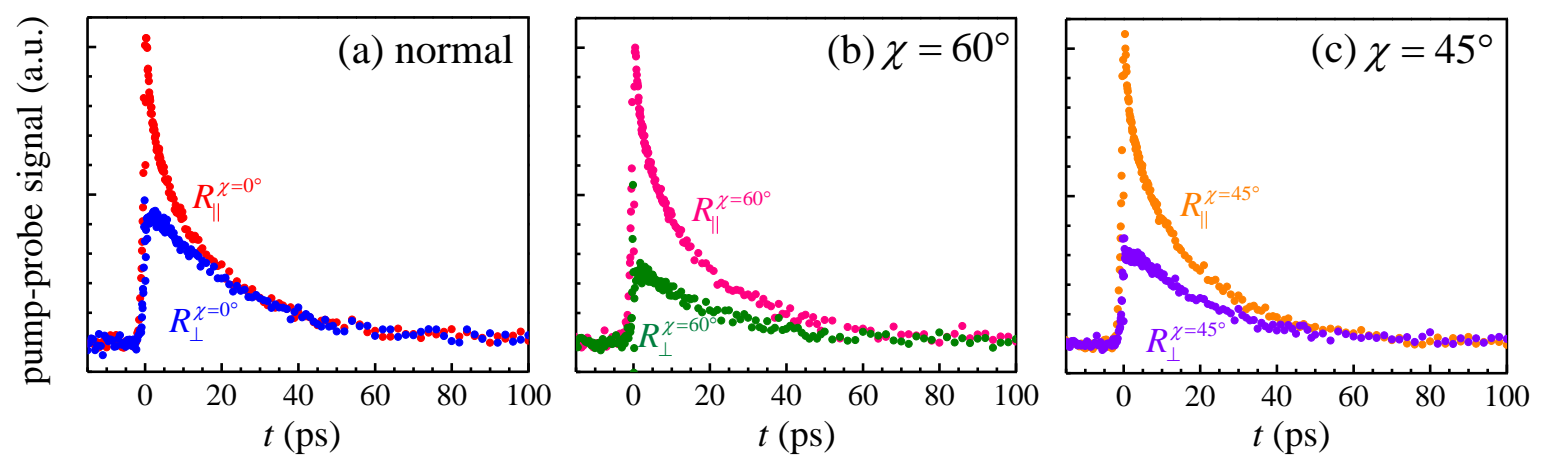

Figure S5. Scaled pump-probe signals acquired for the monolayer in (a) the normal geometry (identical to the data in Fig. 3(a)), (b) the tilted geometry with the tilt angle $60^{\circ}$ (identical to the data in Fig. 3(b)), and (c) the tilted geometry with the tilt angle $45^{\circ}$. Clearly the $45^{\circ}$ data in (c) can be viewed as the data between the normal data in (a) and the $60^{\circ}$ data in (b). The $45^{\circ}$ data can be combined with the normal data to yield the out-of-plane and the mainly-in-plane correlation functions in the same manner applied to the $60^{\circ}$ data in the main text.

perpendicular signals were acquired in the two geometries, namely the "normal" geometry (Fig. 3A) and the "tilted" geometry (Fig. 3B). In the tilted geometry, the tilt angle $\chi$ should be set as large as possible, because the larger tilt angle makes a clearer contrast between the perpendicular signals acquired in the "normal" geometry and the "tilted" geometry. In the main text, we discussed the data acquired with the tilt angle $\chi=60^{\circ}$, because this tilt angle was the largest angle we were able to set in our experimental setup.

Nonetheless, we should obtain the identical $C^{\mathrm{op}}(t)$ and $C^{\mathrm{mip}}(t)$ when we acquired these correlation functions using another tilt angle, as long as the theory in our previous publication ${ }^{6}$ and the local field correction scheme discussed in Section A, including the choice of the effective refractive index $n^{\prime}$, are correct. In this subsection, we will show that $C^{\mathrm{op}}(t)$ and $C^{\mathrm{mip}}(t)$ acquired from the $\chi=45^{\circ}$ is in excellent agreement with the ones obtained from the $\chi=60^{\circ}$ measurement discussed in the main text.

Figure S5 compares the parallel and the perpendicular signals obtained in the normal $\left(\chi=0^{\circ}\right)$ geometry, the tilted geometry with $\chi=60^{\circ}$, and the tilted geometry with $\chi=45^{\circ}$. These signals are scaled such that the parallel signal at $t=0.5 \mathrm{ps}$ is normalized to 1 . Again, the scaled parallel signal in the $\chi=45^{\circ}$ measurement is identical to the parallel signals in the normal and the $\chi=60^{\circ}$ measurements, because all of these signals only involve in-plane $s$-polarized fields. In the main text, the parallel and perpendicular signals in the normal geometry (Fig. S5(a)) and the parallel and perpendicular signals in the tilted geometry with the tilt angle of $\chi=60^{\circ}$ (Fig. $\mathrm{S} 5(\mathrm{~b}))$ were combined to yield $C^{\mathrm{op}}(t)$ and $C^{\text {mip }}(t)$. The same procedure can be followed by 

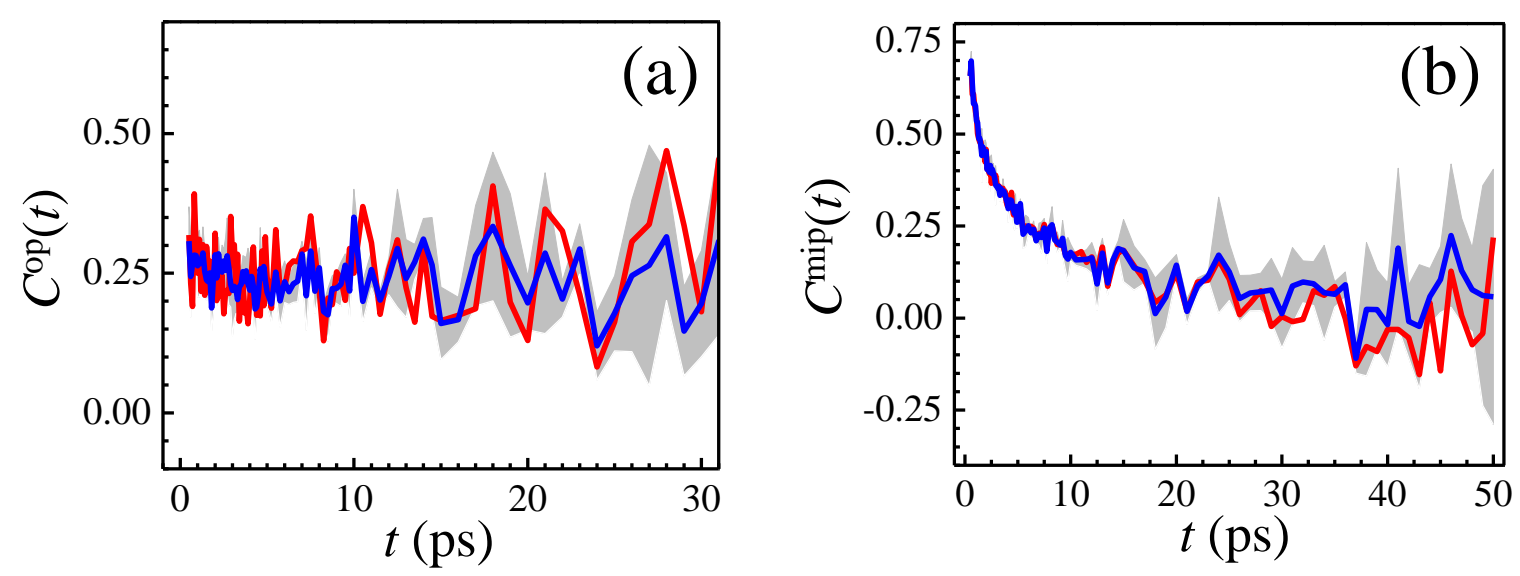

Figure S6. (a) The out-of-plane and (b) the mainly-in-plane correlation functions extracted from the signal with (red) the $\chi=45^{\circ}$ incident angle geometry and (blue) the $\chi=60^{\circ}$ incident angle geometry. The shared normal geometry data was used in the extraction process. The shade is $1 \sigma$ STD error for the $\chi=60^{\circ}$ data. The agreement between the $\chi=45^{\circ}$ data and the $\chi=60^{\circ}$ data is excellent.

using the signals in the normal geometry (Fig. S5(a)) and the tilted geometry with the tilt angle of $\chi=45^{\circ}$ (Fig. S5(c)). $\langle S\rangle=-0.290 \pm 0.016$ can be used as the order parameter, while, for the $\chi=45^{\circ}$ geometry, the effective incident angle is $\chi^{\prime}=37.70^{\circ}$ and the $p / s$ enhancement factor is $a_{p / s}=1.007$ as calculated in Table S1. The results are plotted as red lines in Fig. S6(a) and (b), together with the data from $\chi=60^{\circ}$ measurement as blue lines for comparison. These data agree within the noise and the error, which strongly supports that the theory and the local field correction are both correct, and the choice of the effective refractive index as $n^{\prime}=1.178$ is reasonable. 


\section{E. Orientational Correlation Functions for Numerical Models}

In this section, we will calculate the out-of-plane and the mainly-in-plane correlation functions, and the order parameter for several numerical models.

First, in the following subsection E1, we will briefly review "wobbling-in-a-tilted-cone" model which was developed and discussed in depth in a previous publication (Fig. S7(a)). ${ }^{6}$ Then, we will extend this model to "wobbling-in-a-range-of-tilted-cones" model in E2, where the system is regarded as an ensemble of cones with different tilt angles continuously varying from $\theta_{t, 0}-\Delta \theta_{t}$ to $\theta_{t, 0}+\Delta \theta_{t}$ (Fig. S7(b)). As shown in the main text, this model did not reproduce the observed out-of-plane correlation function well. In E3, we will derive the correlation functions for "wobbling-in-two-cones" model, where the system is assumed to be composed of two kinds of cones with different tilt angles (Fig. S7(c)). This model reproduced the observed out-of-plane correlation function well, and indeed is in accordance with the prediction from a MD simulation on a similar monolayer. ${ }^{14}$

In E4, we will derive the in-plane correlation function for transition dipoles "wobbling-in-asector" (Fig. S7(d)). This is essentially two-dimensional version of widely adopted wobbling-ina-cone model. ${ }^{15}$ This model was necessary to understand the observed mainly-in-plane correlation function decay.
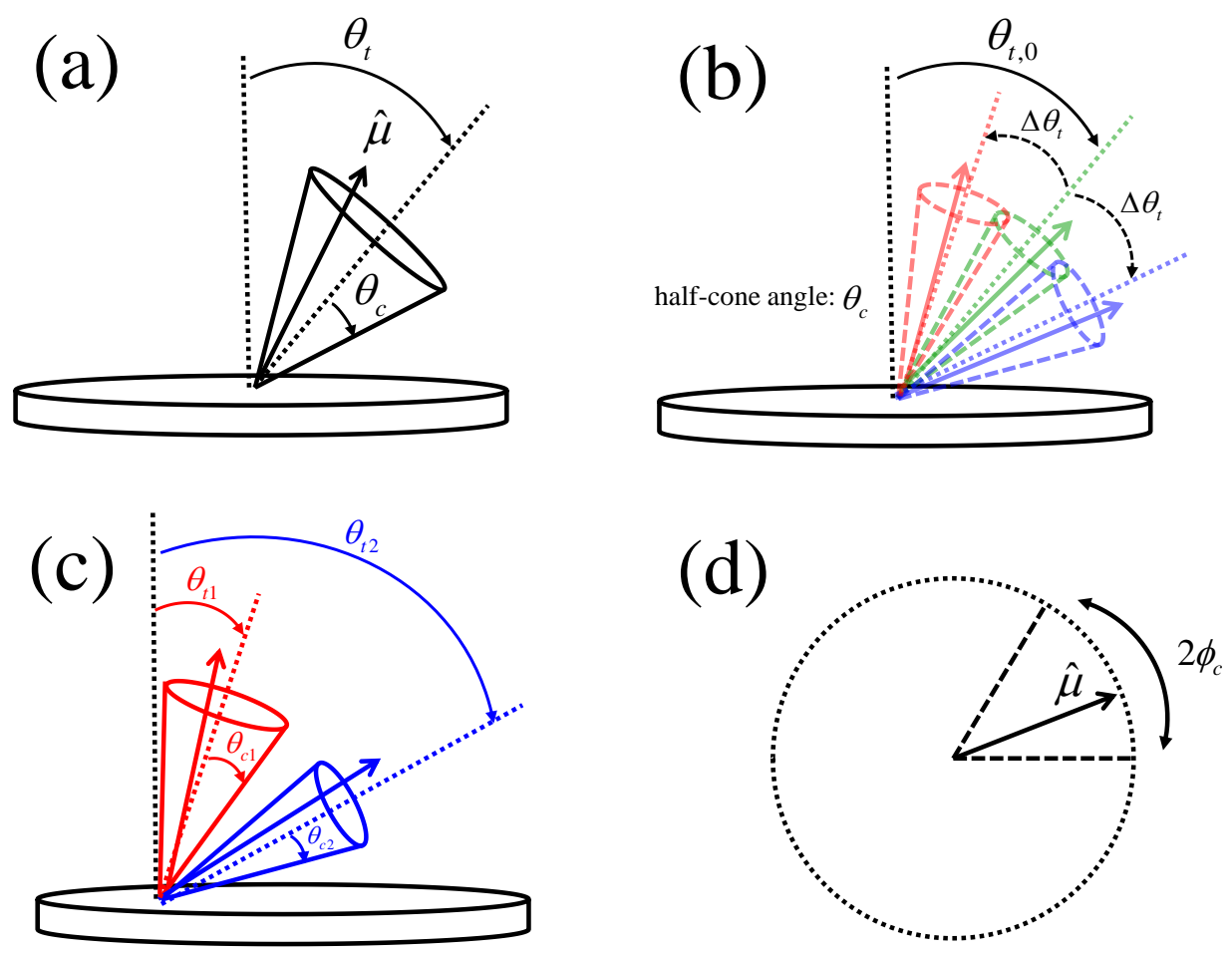

Figure S7. Four numerical models discussed in this section. See the text above and the subsections below for details. 


\section{E1. Wobbling-in-a-tilted-cone Model}

In wobbling-in-a-tilted-cone model (Fig. S7a), we assume the transition dipole is wobbling in a tilted cone with a hard wall. The cone's tilt angle is $\theta_{t}$ and the half-cone angle is $\theta_{c}$. The diffusion constant is $D$. We derived the out-of-plane and the mainly-in-plane correlation functions, and the order parameter for this motion. The detailed derivations are given in the Supporting Information of a previous publication. ${ }^{6}$ The results are accurately approximated up to $\theta_{c} \leq 60^{\circ}$ by:

$$
\begin{aligned}
C_{\theta_{t}, \theta_{c}, D}^{\mathrm{op}}(t)= & \frac{1}{80}\left(3 \cos ^{2} \theta_{t}-1\right)^{2} \times \\
& {\left[5 \cos ^{2} \theta_{c}\left(1+\cos \theta_{c}\right)^{2}+\left(4-\cos \theta_{c}-6 \cos ^{2} \theta_{c}-\cos ^{3} \theta_{c}+4 \cos ^{4} \theta_{c}\right) e^{-v_{2}^{0}\left(v_{2}^{0}+1\right) D t}\right] } \\
+ & \frac{3}{10} \sin ^{2} \theta_{t} \cos ^{2} \theta_{t}\left[2+2 \cos \theta_{c}\left(1+\cos \theta_{c}\right)-3 \cos ^{3} \theta_{c}\left(1+\cos \theta_{c}\right)\right] e^{-v_{1}^{1}\left(v_{1}^{1}+1\right) D t} \\
+ & \frac{3}{160} \sin ^{4} \theta_{t}\left[8-7 \cos \theta_{c}\left(1+\cos \theta_{c}\right)+3 \cos ^{3} \theta_{c}\left(1+\cos \theta_{c}\right)\right] e^{-v_{1}^{2}\left(v_{1}^{2}+1\right) D t}
\end{aligned}
$$

$$
\begin{aligned}
C_{\theta_{t}, \theta_{c}, D}^{\operatorname{mip}}(t)= & \frac{1}{20} \sin ^{4} \theta_{t} \times \\
& {\left[5 \cos ^{2} \theta_{c}\left(1+\cos \theta_{c}\right)^{2}+\left(4-\cos \theta_{c}-6 \cos ^{2} \theta_{c}-\cos ^{3} \theta_{c}+4 \cos ^{4} \theta_{c}\right) e^{-v_{2}^{0}\left(v_{2}^{0}+1\right) D t}\right] } \\
+ & \frac{2}{15} \sin ^{2} \theta_{t}\left(1+\cos ^{2} \theta_{t}\right)\left[2+2 \cos \theta_{c}\left(1+\cos \theta_{c}\right)-3 \cos ^{3} \theta_{c}\left(1+\cos \theta_{c}\right)\right] e^{-v_{1}^{1}\left(v_{1}^{1}+1\right) D t} \\
+ & \frac{1}{15}\left(\sin ^{8} \frac{\theta_{t}}{2}+\cos ^{8} \frac{\theta_{t}}{2}\right)\left[8-7 \cos \theta_{c}\left(1+\cos \theta_{c}\right)+3 \cos ^{3} \theta_{c}\left(1+\cos \theta_{c}\right)\right] e^{-v_{1}^{2}\left(v_{1}^{2}+1\right) D t}
\end{aligned}
$$

$$
\langle S\rangle_{\theta_{t}, \theta_{c}, D}=\frac{\left(3 \cos ^{2} \theta_{t}-1\right) \cos \theta_{c}\left(1+\cos \theta_{c}\right)}{4}
$$

While there are several approximations provided for $v_{2}^{0}, v_{1}^{1}$ and $v_{1}^{2},{ }^{16,17}$ we re-evaluated these factors as a numerical solution of an equation provided by Wang and Pecora, ${ }^{15}$ and found that these are well approximated by $\left(10^{\circ} \leq \theta_{c} \leq 90^{\circ}\right)$

$$
\begin{aligned}
& v_{2}^{0}\left(v_{2}^{0}+1\right) \approx \frac{14.68}{\theta_{c}^{2}} \\
& v_{1}^{1}\left(v_{1}^{1}+1\right) \approx \frac{24}{7 \theta_{c}^{2}}\left(0.98+0.056 \theta_{c}+0.029 \theta_{c}^{2}+0.077 \theta_{c}^{3}\right)
\end{aligned}
$$




$$
v_{1}^{2}\left(v_{1}^{2}+1\right) \approx \frac{9.456}{\theta_{c}^{2}}\left(0.973+0.0878 \theta_{c}-0.00376 \theta_{c}^{2}+0.11875 \theta_{c}^{3}\right)
$$

Note that this model cannot capture the observed $C^{\mathrm{op}}(t)$, because in this model, after long enough time, $C^{\text {op }}(t)$ decays to $\langle S\rangle^{2}$ :

$$
C^{\mathrm{op}}(\infty)=\frac{\left(3 \cos ^{2} \theta_{t}-1\right)^{2} \cos ^{2} \theta_{c}\left(1+\cos \theta_{c}\right)^{2}}{16}=\langle S\rangle^{2}
$$

As discussed in the main text, this is not what we are observing in Fig. $5 \mathrm{~A}$, and $C^{\mathrm{op}}(\infty)$ is significantly higher than the experimental $\langle S\rangle^{2}$, indicating that the transition dipoles are not sampling all the possible configurations in the time window of the experiment.

The results in Eqs. (S.25) are important for the following calculations in E2 and E3.

\section{E2. Wobbling-in-a-range-of-tilted-cones Model}

To reproduce the observed $C^{\mathrm{op}}(t)$ which is significantly higher than $\langle S\rangle^{2}$ even after long time, we first calculated the correlation functions and the order parameter for transition dipoles which are wobbling with the identical diffusion constant $D$ and cone angle $\theta_{c}$, but the tilt angles of the cones vary continuously from $\theta_{t, 0}-\Delta \theta_{t}$ to $\theta_{t, 0}+\Delta \theta_{t}$ as shown in Fig. S7(b). The correlation functions and the order parameter for this case can be calculated as

$$
\begin{gathered}
C_{\text {range }}^{\text {op }}(t)=\frac{\int_{\theta_{t, 0}-\Delta \theta_{t}}^{\theta_{t, 0}+\Delta \theta_{t}} d \theta_{t} \sin \theta_{t} \times C_{\theta_{t}, \theta_{c}, D}^{\text {op }}(t)}{\int_{\theta_{t, 0}-\Delta \theta_{t}}^{\theta_{t, 0} \Delta \theta_{t}} d \theta_{t} \sin \theta_{t}} \\
C_{\text {range }}^{\text {mip }}(t)=\frac{\int_{\theta_{t, 0}-\Delta \theta_{t}}^{\theta_{t,}+\Delta \theta_{t}} d \theta_{t} \sin \theta_{t} \times C_{\theta_{t}, \theta_{c}, D}^{\text {mip }}(t)}{\int_{\theta_{t, 0}-\Delta \theta_{t}}^{\theta_{t, 0} \Delta \theta_{t}} d \theta_{t} \sin \theta_{t}} \\
\langle S\rangle_{\text {range }}=\frac{\int_{\theta_{t, 0}-\Delta \theta_{t}}^{\theta_{t, 0}+\Delta \theta_{t}} d \theta_{t} \sin \theta_{t} \times\langle S\rangle_{\theta_{t}, \theta_{c}, D}}{\int_{\theta_{t, 0}-\Delta \theta_{t}}^{\theta_{t,} \Delta \theta_{t}} d \theta_{t} \sin \theta_{t}}
\end{gathered}
$$

where $C_{\theta_{t}, \theta_{c}, D}^{\mathrm{op}}, C_{\theta_{t}, \theta_{c}, D}^{\mathrm{mip}}$ and $\langle S\rangle_{\theta_{t}, \theta_{c}, D}$ are given by Eqs. (S.25a)-(S.25c). Note that all the integrals can be calculated analytically, and thus (S.28a)-(S.28c) have well-defined analytical forms given by: 


$$
\begin{aligned}
C_{\theta_{t}, \theta_{c}, D}^{\mathrm{op}}(t)= & \frac{1}{2 \sin \left(\Delta \theta_{t}\right) \sin \left(\theta_{t, 0}\right)} \times \frac{1}{6400} \times \\
& \left\{2 \times\left\{50 \sin \left(\Delta \theta_{t}\right) \sin \left(\theta_{t, 0}\right)+5 \sin \left(3 \Delta \theta_{t}\right) \sin \left(3 \theta_{t, 0}\right)+9 \sin \left(5 \Delta \theta_{t}\right) \sin \left(5 \theta_{t, 0}\right)\right\}\right. \\
& \times\left[5 \cos ^{2} \theta_{c}\left(1+\cos \theta_{c}\right)^{2}+\left(4-\cos \theta_{c}-6 \cos ^{2} \theta_{c}-\cos ^{3} \theta_{c}+4 \cos ^{4} \theta_{c}\right) e^{-v_{2}^{0}\left(v_{2}^{0}+1\right) D t}\right] \\
+ & +\left\{30 \sin \left(\Delta \theta_{t}\right) \sin \left(\theta_{t, 0}\right)+5 \sin \left(3 \Delta \theta_{t}\right) \sin \left(3 \theta_{t, 0}\right)-3 \sin \left(5 \Delta \theta_{t}\right) \sin \left(5 \theta_{t, 0}\right)\right\} \\
& \times\left[2+2 \cos \theta_{c}\left(1+\cos \theta_{c}\right)-3 \cos ^{3} \theta_{c}\left(1+\cos \theta_{c}\right)\right] e^{-v_{1}^{1}\left(v_{1}^{1}+1\right) D t} \\
+ & \left\{150 \sin \left(\Delta \theta_{t}\right) \sin \left(\theta_{t, 0}\right)-25 \sin \left(3 \Delta \theta_{t}\right) \sin \left(3 \theta_{t, 0}\right)+3 \sin \left(5 \Delta \theta_{t}\right) \sin \left(5 \theta_{t, 0}\right)\right\} \\
& \left.\times\left[8-7 \cos \theta_{c}\left(1+\cos \theta_{c}\right)+3 \cos ^{3} \theta_{c}\left(1+\cos \theta_{c}\right)\right] e^{-v_{1}^{2}\left(v_{1}^{2}+1\right) D t}\right\}
\end{aligned}
$$

$$
\begin{aligned}
C_{\theta_{t}, \theta_{c}, D}^{\operatorname{mip}}(t)= & \frac{1}{2 \sin \left(\Delta \theta_{t}\right) \sin \left(\theta_{t, 0}\right)} \times \frac{1}{4800} \times \\
& \left\{2 \times\left\{150 \sin \left(\Delta \theta_{t}\right) \sin \left(\theta_{t, 0}\right)-25 \sin \left(3 \Delta \theta_{t}\right) \sin \left(3 \theta_{t, 0}\right)+3 \sin \left(5 \Delta \theta_{t}\right) \sin \left(5 \theta_{t, 0}\right)\right\}\right.
\end{aligned}
$$$$
\times\left[5 \cos ^{2} \theta_{c}\left(1+\cos \theta_{c}\right)^{2}+\left(4-\cos \theta_{c}-6 \cos ^{2} \theta_{c}-\cos ^{3} \theta_{c}+4 \cos ^{4} \theta_{c}\right) e^{-v_{2}^{0}\left(v_{2}^{0}+1\right) D t}\right]
$$$$
+16 \times\left\{70 \sin \left(\Delta \theta_{t}\right) \sin \left(\theta_{t, 0}\right)-5 \sin \left(3 \Delta \theta_{t}\right) \sin \left(3 \theta_{t, 0}\right)-\sin \left(5 \Delta \theta_{t}\right) \sin \left(5 \theta_{t, 0}\right)\right\}
$$$$
\times\left[2+2 \cos \theta_{c}\left(1+\cos \theta_{c}\right)-3 \cos ^{3} \theta_{c}\left(1+\cos \theta_{c}\right)\right] e^{-v_{1}^{1}\left(v_{1}^{1}+1\right) D t}
$$$$
+\left\{210 \sin \left(\Delta \theta_{t}\right) \sin \left(\theta_{t, 0}\right)+45 \sin \left(3 \Delta \theta_{t}\right) \sin \left(3 \theta_{t, 0}\right)+\sin \left(5 \Delta \theta_{t}\right) \sin \left(5 \theta_{t, 0}\right)\right\}
$$$$
\left.\times\left[8-7 \cos \theta_{c}\left(1+\cos \theta_{c}\right)+3 \cos ^{3} \theta_{c}\left(1+\cos \theta_{c}\right)\right] e^{-v_{1}^{2}\left(v_{1}^{2}+1\right) D t}\right\}
$$

$$
\langle S\rangle_{\theta_{t}, \theta_{c}, D}=\frac{1}{16} \cdot \frac{-\sin \left(\Delta \theta_{t}\right) \sin \theta_{t, 0}+\sin \left(3 \Delta \theta_{t}\right) \sin 3 \theta_{t, 0}}{\sin \left(\Delta \theta_{t}\right) \sin \theta_{t, 0}} \cdot \cos \theta_{c}\left(1+\cos \theta_{c}\right)
$$

The out-of-plane correlation function in Eq. (S.29a) was used to fit the data in Fig. 5A by varying $\theta_{c}, \theta_{t, 0}, \Delta \theta_{t}$ and $D$, under the condition that the order parameter in Eq. (S.29c) yields the observed order parameter of $\langle S\rangle=-0.290 \pm 0.016$. As shown as a green line in Fig. 5A, this model was not able to reproduce the observed out-of-plane correlation function together with the observed order parameter.

\section{E3. Wobbling-in-two-cones Model}

In wobbling-in-two-cones model, we assume that the transition dipoles are wobbling in either one of two types of cones (Fig. S7(c)). The tilt angle, the cone angle, and the diffusion constant 
are all independent for the two cones. The correlation functions and the order parameter for this case can be written as:

$$
\begin{aligned}
& C_{\text {two cones }}^{\mathrm{op}}(t)=\frac{N_{1} C_{\theta_{t 1}, \theta_{c 1}, D_{1}}^{\mathrm{op}}(t)+N_{2} C_{\theta_{t 2}, \theta_{c 2}, D_{2}}^{\mathrm{op}}(t)}{N_{1}+N_{2}} \\
& C_{\text {two cones }}^{\text {mip }}(t)=\frac{N_{1} C_{\theta_{t 1}, \theta_{c 1}, D_{1}}^{\mathrm{mip}}(t)+N_{2} C_{\theta_{t 2}, \theta_{c 2}, D_{2}}^{\mathrm{mip}}(t)}{N_{1}+N_{2}} \\
& \langle S\rangle_{\text {two cones }}=\frac{N_{1}\langle S\rangle_{\theta_{t 1}, \theta_{c 1}, D_{1}}+N_{2}\langle S\rangle_{\theta_{t 2}, \theta_{c 2}, D_{2}}}{N_{1}+N_{2}},
\end{aligned}
$$

where $C_{\theta_{t}, \theta_{c}, D}^{\mathrm{op}}(t), C_{\theta_{t}, \theta_{c}, D}^{\mathrm{op}}(t)$ and $\langle S\rangle_{\theta_{t}, \theta_{c}, D}$ are those calculated from Eqs. (S.25) for a single cone model. $N_{1}$ and $N_{2}$ are the surface densities of the transition dipoles wobbling in cone 1 and cone 2 , respectively. What matters here is the ratio of the density $N_{1} / N_{2}$ rather than their absolute values.

Again, the out-of-plane correlation function in Fig. 5A was fit with Eqs. (S.30a) so that the order parameter calculated based on Eq. (S.30c) yields the observed order parameter $\langle S\rangle=-0.290 \pm 0.016$. As discussed in the main text, this model fits the observed correlation function and reproduced the order parameter fairly well. Based on the fitting parameters obtained from the out-of-plane correlation function, the contribution of the two-cone wobbling motion in the mainly-in-plane correlation function can be calculated by Eq. (S.30b). The observed mainlyin-plane correlation function decays more significantly than the two-cone motion, indicating that there exists significant in-plane motion on the top of this two-cone motion.

\section{E4. Wobbling-in-a-sector Model}

In this subsection, we will discuss a transition dipole reorienting on a strictly two-dimensional plane. This is equivalent of a transition dipole moment in a three-dimensional space with a completely frozen polar motion and a free or restricted azimuthal motion.

Restricted Two-dimensional Rotation. We are particularly interested in a transition dipole wobbling in a "sector" (Fig. S7(d)) with a full sector angle $2 \phi_{c}$ with a diffusion constant $D$. For convenience, we assume that the center of the sector is located at $\phi=0$, and the hard walls are located at $\phi=-\phi_{c}$ and $\phi=+\phi_{c}$. We will calculate the in-plane correlation function $C^{\mathrm{ip}}(t)=\left\langle e^{2 i \phi(t)}\left(e^{2 i \phi(0)}\right)^{*}\right\rangle$ for such a transition dipole, eventually given in Eq. (S.41). A transition dipole wobbling in a sector centered at $\phi \neq 0$ yields an identical result. 
The probability $W(\phi, t)$ that a transition dipole is pointing toward $\phi$ at time $t$ obeys the following differential equation and the boundary condition:

$$
\begin{aligned}
& \frac{\partial W(\phi, t)}{\partial t}=D \frac{\partial^{2} W(\phi, t)}{\partial \phi^{2}} \\
& \left.\frac{\partial W(\phi, t)}{\partial \phi}\right|_{\phi= \pm \phi_{c}}=0
\end{aligned}
$$

which yields the general solution of $W(\phi, t)$ in the form of

$$
W(\phi, t)=\sum_{n=0}^{+\infty} c_{n} \times \exp \left[-D\left(\pi n / 2 \phi_{c}\right)^{2} t\right] \times f_{n}(\phi)
$$

where $f_{n}(\phi)$ is given by

$$
f_{n}(\phi)=\exp \left[i \frac{\pi n}{2 \phi_{c}} \phi\right]+(-1)^{n} \exp \left[-i \frac{\pi n}{2 \phi_{c}} \phi\right]
$$

The orthogonality of $f_{n}(\phi)$ can be easily verified:

$$
\int_{\phi=-\phi_{c}}^{\phi=+\phi_{c}} f_{n}(\phi) f_{m}^{*}(\phi)=\left\{\begin{array}{cc}
0 & (n \neq m) \\
8 \phi_{c} & (n=m=0) \\
4 \phi_{c} & (n=m>0)
\end{array}\right.
$$

Because the transition dipole must be found somewhere between $\phi=-\phi_{c}$ and $\phi=+\phi_{c}, W(\phi, t)$ must satisfy

$$
\int_{-\phi_{c}}^{+\phi_{c}} d \phi W(\phi, t)=1
$$

for any time $t . c_{n}$ in Eq. (S.32) depends on the initial distribution of the transition dipoles. Particularly for $W(\phi, 0)=\delta\left(\phi-\phi_{0}\right)$, which obviously satisfies Eq. (S.35), $c_{n}$ is given by

$$
c_{n}= \begin{cases}\frac{1}{8 \phi_{c}} f_{0}^{*}\left(\phi_{0}\right) & (n=0) \\ \frac{1}{4 \phi_{c}} f_{n}^{*}\left(\phi_{0}\right) & (n>0)\end{cases}
$$



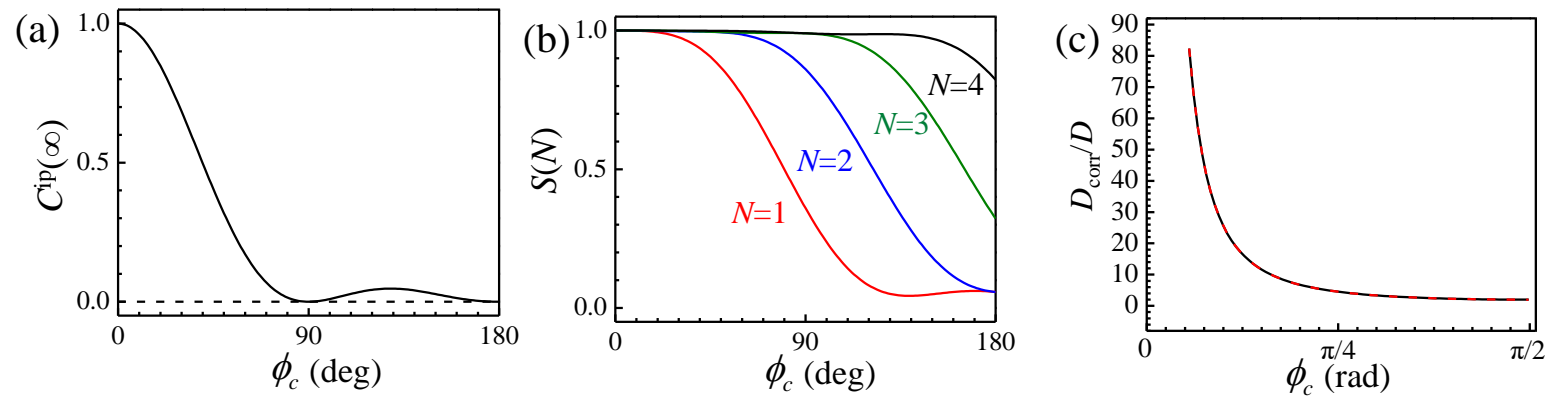

Figure S8. Plots related to the "wobbling-in-a-sector" model. (a) The plot of $C^{\text {ip }}(\infty)=\left[\operatorname{sinc}\left(2 \phi_{c}\right)\right]^{2}$ with respect to the half-cone angle $\phi_{c}$. (b) $S(N)$ in Eq. (S.39) for $N=1,2,3$ and 4 with respect to the half-cone angle $\phi_{c}$. The value close to 1 indicates that the exact infinite summation is well-approximated by the summation up to the $N$ th term. (c) $D_{\text {corr }} / D$ with respect to the half-cone angle $\phi_{c}$ in radian. $D_{\text {corr }}$ was calculated as $1 / \tau_{\text {corr }}$, where $\tau_{\text {corr }}$ was calculated based on Eq. (S.40). $C^{\text {ip }}(t)$ was calculated as in Eq. (S.38) with the summation taken up to $4^{\text {th }}$ term, which should be an excellent approximation based on the plot in (b).

$W(\phi, t)$ obtained above with the initial distribution $W(\phi, 0)=\delta\left(\phi-\phi_{0}\right)$ can be regarded as a Green's function $G\left(\phi, t \mid \phi_{0}, 0\right)$, which corresponds to the probability that we find a transition dipole moment pointing toward $\phi_{0}$ at time $t=0$ and pointing toward $\phi$ at later time $t$ :

$$
G\left(\phi, t \mid \phi_{0}, 0\right)=\frac{1}{2 \phi_{c}}+\frac{1}{4 \phi_{c}} \sum_{n=1}^{\infty} e^{-D\left(\pi n / 2 \phi_{c}\right)^{2} t} f_{n}(\phi) f_{n}^{*}\left(\phi_{0}\right)
$$

Now that we obtain the form of the Green's function, we can calculate the correlation function $C^{\mathrm{ip}}(t)=\left\langle e^{2 i \phi(t)}\left(e^{2 i \phi(0)}\right)^{*}\right\rangle$. At $t=0$, the transition dipole moments must be equally distributed between $\phi=-\phi_{c}$ and $\phi=+\phi_{c}$, i.e. $W(\phi, 0)=\frac{1}{2 \phi_{c}}$, which satisfies Eq. (S.35). Based on this initial distribution and the Green's function in Eq. (S.37), the correlation function can be calculated as

$$
\begin{aligned}
\left\langle e^{2 i \phi(t)}\left(e^{2 i \phi(0)}\right)^{*}\right\rangle= & \int_{-\phi_{c}}^{+\phi_{c}} d \phi \int_{-\phi_{c}}^{+\phi_{c}} d \phi_{0} e^{2 i \phi} G\left(\phi, t \mid \phi_{0}, 0\right) e^{-2 i \phi_{0}} W\left(\phi_{0}, 0\right) \\
= & \frac{1}{2 \phi_{c}} \int_{-\phi_{c}}^{+\phi_{c}} d \phi \int_{-\phi_{c}}^{+\phi_{c}} d \phi_{0} e^{2 i \phi}\left(\frac{1}{2 \phi_{c}}+\frac{1}{4 \phi_{c}} \sum_{n=1}^{\infty} e^{-D\left(\pi n / 2 \phi_{c}\right)^{2} t} f_{n}(\phi) f_{n}^{*}\left(\phi_{0}\right)\right) e^{-2 i \phi_{0}} \\
= & \frac{1}{4 \phi_{c}^{2}}\left|\int_{-\phi_{c}}^{+\phi_{c}} d \phi e^{2 i \phi}\right|^{2} \\
& +\frac{1}{8 \phi_{c}^{2}} \sum_{n=1}^{\infty} e^{-D\left(\pi n / 2 \phi_{c}\right)^{2} t} \int_{-\phi_{c}}^{+\phi_{c}} d \phi \int_{-\phi_{c}}^{+\phi_{c}} d \phi_{0} e^{2 i \phi} f_{n}(\phi) f_{n}^{*}\left(\phi_{0}\right) e^{-2 i \phi_{0}} \\
= & {\left[\operatorname{sinc}\left(2 \phi_{c}\right)\right]^{2}+\frac{1}{2} \sum_{n=1}^{\infty} e^{-D\left(\pi n / 2 \phi_{c}\right)^{2} t}\left\{\operatorname{sinc}\left(2 \phi_{c}+\pi n / 2\right)+(-1)^{n} \operatorname{sinc}\left(2 \phi_{c}-\pi n / 2\right)\right\}^{2} }
\end{aligned}
$$


Note that $\operatorname{sinc}$ function here is defined as $\operatorname{sinc}(x) \equiv \sin x / x$. Eq. (S.38) indicates that $C^{\mathrm{ip}}(t)$ decays as the sum of infinite number of exponentials, while, as we will show later, taking just the first $n=1$ term in the summation is a very good approximation to the exact infinite sum, and the summation up to $n=2$ is almost the perfect approximation when $\phi_{c} \leq 50^{\circ}$. The offset in this correlation decay obtained as $t \rightarrow \infty$ is exactly $\left[\operatorname{sinc}\left(2 \phi_{c}\right)\right]^{2}$, which is plotted in Fig. S8(a). At $\phi_{c}=0^{\circ}$, the transition dipole cannot reorient at all, so the $C^{\mathrm{ip}}(t)$ stays at its maximum possible value of 1 and never decays. As the half-sector angle $\phi_{c}$ becomes large, the offset decreases and at $\phi_{c}=90^{\circ}$ (full-sector angle of $180^{\circ}$ ) there is no offset. There is a minor recurrence for $\phi_{c}>90^{\circ}$, though such a situation as $\phi_{c}>90^{\circ}$ may not be physically realistic.

Note that at $t=0$, regardless of the sector angle, the transition dipoles have not moved and therefore $C^{\text {ip }}(0)=1$. Here we define

$$
S(N)=\left(\operatorname{sinc}\left[2 \phi_{c}\right]\right)^{2}+\frac{1}{2} \sum_{n=1}^{N}\left\{\operatorname{sinc}\left(2 \phi_{c}+\pi n / 2\right)+(-1)^{n} \operatorname{sinc}\left(2 \phi_{c}-\pi n / 2\right)\right\}^{2}
$$

Note that $S(\infty)=C^{\mathrm{ip}}(0)=1$. Thus, instead of taking the infinite summation in Eq. (S.38), the summation can be taken up to such $N$ that makes $S(N)$ approximately $1 . S(N)$ with respect to the half-sector angle $\phi_{c}$ for various $N$ is given in Fig. S8(b). It can be seen that $N=2$, i.e. the summation up to the second term, almost perfectly approximates the exact infinite summation in a physically realistic situation of $\phi_{c} \leq 90^{\circ}$. If $\phi_{c} \leq 60^{\circ}$, even if we take only the first term it is indeed a fairly good approximation.

It is useful to calculate the correlation time defined as

$$
\tau_{\text {corr }}=\frac{\int_{0}^{\infty}\left(C^{\mathrm{ip}}(t)-C^{\mathrm{ip}}(\infty)\right) d t}{1-C^{\mathrm{ip}}(\infty)}
$$

The correlation time provides a good approximation to the $C^{\mathrm{ip}}(t)$ by

$$
C^{\mathrm{ip}}(t) \approx\left[\operatorname{sinc}\left(2 \phi_{c}\right)\right]^{2}+\left(1-\left[\operatorname{sinc}\left(2 \phi_{c}\right)\right]^{2}\right) \exp \left(-t / \tau_{\text {corr }}\right)
$$

We are interested in how the actual diffusion constant $D$ in Eq. (S.31a) is related to the decay rate in $C^{\text {ip }}(t)$. Fig. S $8\left(\right.$ c) plots $D_{\text {corr }}=\frac{1}{\tau_{\text {corr }}}$ normalized by the diffusion constant $D$ with respect to the half-sector angle $\phi_{c}$ from $10^{\circ}$ to $90^{\circ}$. It turns out that $D_{\text {corr }}$ is well approximated by

$$
D_{\text {corr }} \approx \frac{2.77}{\phi_{c}^{1.94}}\left(1.09-0.63 \phi_{c}+1.23 \phi_{c}^{2}-0.96 \phi_{c}^{3}+0.38 \phi_{c}^{4}\right) D
$$

where $\phi_{c}$ is given in radian. Thus to extract $D$, the following procedure should be taken: 
(1) First, based on the offset level $C^{\text {ip }}(\infty)=\left[\operatorname{sinc}\left(2 \phi_{c}\right)\right]^{2}, \phi_{c}$ is obtained.

(2) Based on the observed time constant $\tau_{\text {obs }}, D_{\text {obs }}=1 / \tau_{\text {obs }}$ can be calculated. $D_{\text {obs }}$ corresponds to $D_{\text {corr }}$ in Eq. (S.42). Based on the known $\phi_{c}, D$ can be acquired by solving Eq. (S.42).

\section{Free Two-dimensional Rotation.}

In case the transition dipoles are reorienting in two-dimensional plane without any restrictions (free rotation), the same procedure above can be followed by replacing the boundary condition in Eq. (S.31b) with

$$
W(\phi, t)=W(\phi+2 \pi, t)
$$

which eventually yields

$$
C^{\mathrm{ip}}(t)=\exp (-4 D t)
$$

The identical result was previously obtained by Bonn and co-workers. ${ }^{18}$ Because there is no restriction on the motion of the transition dipole moment, $C^{\text {ip }}(t)$ decays to zero at long time. 


\section{F. Förster Excitation Transfer}

In the main text we attribute the decay in the orientational correlation functions $C^{\mathrm{op}}(t)$ and $C^{\text {mip }}(t)$ to the reorientation of the transition dipoles; the decay could also be caused by Förster excitation transfer. In Förster excitation transfer, the excitation on a particular molecule is transferred to molecule through a transition dipole-transition dipole interaction. When the directions of the two transition dipoles involved are different, this process leads to the decay of the orientational correlation functions. The rate of the excitation transfer depends both on the strength of the transition dipoles involved, and the distance between the dipoles. It also depends on the overlap of the vibrational homogeneous lines of the donor molecule and the acceptor molecule. ${ }^{19}$ Two molecules can be close in space but have little overlap between their homogeneous lines, resulting in very slow excitation transfer. In the monolayer sample studied here, we are studying the vibrational probe with a very large transition dipole and the distance between the transition dipoles is relatively close $(\sim 7.5 \AA)$, so we need to be cautious about the possible contribution of Förster excitation transfer in the observed $C^{\text {op }}(t)$ and $C^{\text {mip }}(t)$ decays.

Förster excitation transfer is expected to contribute to spectral diffusion decay measured in twodimensional infrared spectroscopy as well; when the two transition dipoles involved in the excitation transfer are oscillating with different frequencies, the excitation transfer leads to the decay in the frequency-frequency correlation function. It was found for the type of samples studied here that the spectral diffusion rate is insensitive to the density of the head groups on the surface, indicating that the Förster excitation transfer is indeed negligible in this monolayer. ${ }^{9}$ This absence of the excitation transfer process, regardless of the strong transition dipole and the high density, can be explained by the strongly inhomogeneously broadened infrared absorption band of the symmetric $\mathrm{CO}$ stretching mode. As experimentally derived from two-dimensional infrared spectroscopy, out of $\sim 16 \mathrm{~cm}^{-1}$ fwhm absorption band, the homogeneous line width is less than $2 \mathrm{~cm}^{-1} \mathrm{fwhm} .{ }^{9}$ For the excitation transfer to take place, the frequencies of the two transition dipoles must overlap, which is unlikely due to the small homogeneous/inhomogeneous line width ratio. Thus the strongly inhomogeneously broadened band is preventing the excitation transfer.

These previous studies by two-dimensional infrared spectroscopy demonstrated that the Förster excitation transfer is absent in this sample, but it is important to directly verify that the contribution from Förster excitation transfer is minor in our current observables of the orientational correlation functions. First, we will show that the excitation transfer induced depolarization does not reproduce the time-dependence of the 2D anisotropy well (Fig. 9A). Second, we will show that the observed 2D anisotropy decay rate is less sensitive to the loading of the head groups than expected from the excitation transfer induced depolarization.

We will compare so called two-dimensional anisotropy defined as

$$
r^{2 \mathrm{D}}(t)=\frac{R_{\|}^{\chi=0^{\circ}}(t)-R_{\perp}^{\chi=0^{\circ}}(t)}{R_{\|}^{\chi=0^{\circ}}(t)+R_{\perp}^{\chi=0^{\circ}}(t)},
$$



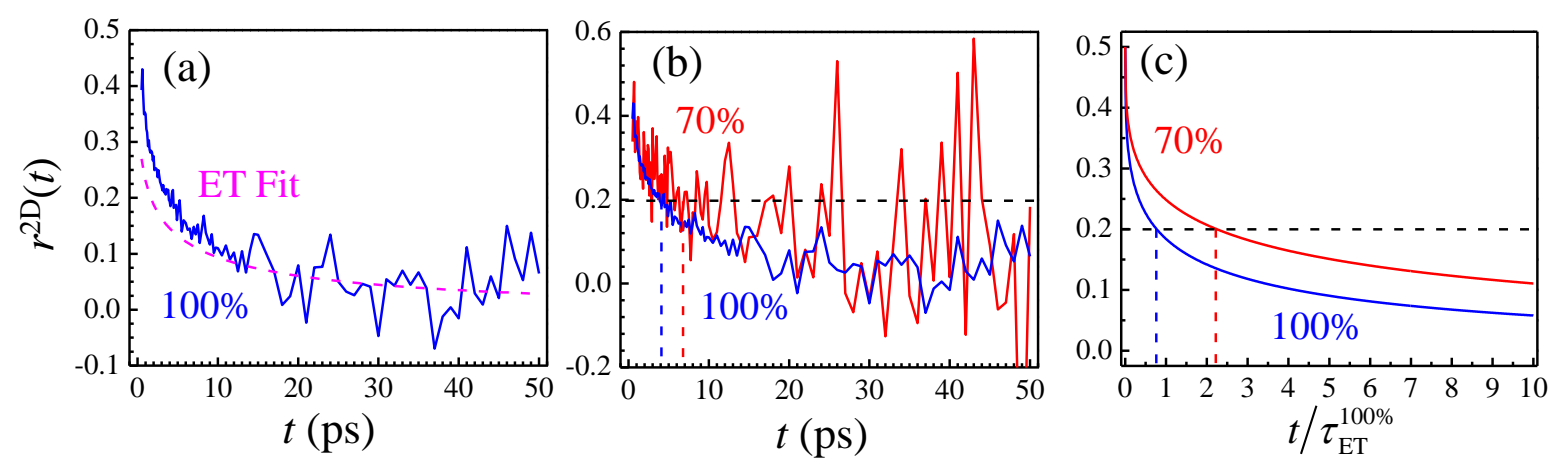

Fig. S9 (a) The attempt to fit the observed 2D anisotropy decay for the 100\% loading sample with the excitation transfer induced depolarization decay in Eq. (S.46). The fit clearly does not reproduce the observed data well. (b) The loading dependence of the 2D anisotropy decay. It is hard to discern these two decays are indeed different. (c) The loading dependence of the 2D anisotropy decay arising from the excitation transfer induced depolarization. While the two plots in (b) could be indeed different, the difference is smaller than the calculated plots in (c), supporting that the excitation transfer induced depolarization cannot be a dominant depolarization mechanism.

which can be obtained from the normal geometry measurement. This $2 \mathrm{D}$ anisotropy is proportional to the in-plane correlation function if the in-plane and out-of-plane motions of the transition dipoles are decoupled. This decoupling of the two motions should be a fairly good approximation in our sample because we demonstrated that the out-of-plane dynamics is strongly restricted while there is a significant in-plane dynamics. In case the excitation transfer is the dominant mechanism of the orientational correlation function decay, 2D anisotropy experimentally obtained through Eq. (S.45) should decay as ${ }^{20}$

$$
r^{2 \mathrm{D}}(t)=\frac{1}{2} e^{-\left(t / \tau_{\mathrm{ET}}\right)^{1 / 3}}
$$

where $\tau_{\mathrm{ET}}$ is inversely proportional to the cube of the transition dipole density $\rho: \tau_{\mathrm{ET}} \propto \rho^{-3}$, which makes the decay in Eq. (S.46) extremely sensitive to the loading of the vibrational probe.

The solid line in Fig. S9(a) shows the 2D anisotropy for the sample discussed in the main text (100\% loading sample). We attempted to fit this data with Eq. (S.46), and the dotted line in Fig. S9(a) is the best fit, which clearly is not reproducing the decay curve. Excitation transfer induced depolarization is not the mechanism causing the 2D anisotropy decay.

Fig. S9(b) shows $r^{2 \mathrm{D}}(t)$ for the $100 \%$ loading sample and the $70 \%$ loading sample (for the synthesis procedure, see Sec. B). The data for $70 \%$ loading sample is much noisier because of the lower signal level and the less averaging. Though it is unclear if these two decays are distinctly different beyond the noise level, admittedly it appears that the decay in the $70 \%$ sample is consistently higher than the decay in the $100 \%$ loading sample. Nonetheless, this difference is much smaller than what we expect from the excitation transfer induced depolarization. The plots in Fig. S9(c) are the calculated 2D anisotropy decay based on Eq. (S.46) for the $100 \%$ loading sample and the $70 \%$ loading sample. The time axis is normalized by $\tau_{\mathrm{ET}}$ for the $100 \%$ loading 
sample. As seen in Fig. S9(c), in the case of the excitation transfer induced depolarization, it takes roughly 3 times longer for the 70\% loading sample to reach the 2D anisotropy level of 0.2 than the $100 \%$ loading sample. The observed 2D anisotropies for the $100 \%$ and the $70 \%$ samples reach 0.2 around $t=4 \mathrm{ps}$ and $7 \mathrm{ps}$ respectively, which is much closer to each other than what is expected from the excitation transfer process.

Based on these two observations, together with the previous results from two-dimensional infrared spectroscopy, we conclude that the orientational correlation function decays are caused by the reorientation of the transition dipoles as discussed in the main text rather than the intermolecular Förster excitation transfer process.

The small difference between the decay in the 100\% sample and the 70\% sample in Fig. S9(b), if it is real, may be attributed to the actual difference in the structures and dynamics between the two samples. Indeed, the absorption band center position for the $70 \%$ sample was $\sim 2 \mathrm{~cm}^{-1}$ blue shifted compared with the $100 \%$ sample, indicating that the structure of the monolayer is affected by the loading of the head group. Also, for the monolayer on a gold surface, it has been shown that the lower-loading monolayer shows slower dynamics, which was verified by both a two-dimensional infrared spectroscopy measurement and a MD simulation. ${ }^{14}$ 


\section{References}

(1) Shen, Y. Annu. Rev. Phys. Chem. 1989, 40, 327.

(2) Heinz, T. F. Nonlinear Surface Electromagnetic Phenomena 1991, 29.

(3) Yamaguchi, S.; Hosoi, H.; Yamashita, M.; Sen, P.; Tahara, T. J. Phys. Chem. Lett. 2010, $1,2662$.

(4) Zhuang, X.; Miranda, P. B.; Kim, D.; Shen, Y. R. Phys. Rev. B 1999, 59, 12632.

(5) Kischkat, J.; Peters, S.; Gruska, B.; Semtsiv, M.; Chashnikova, M.; Klinkmüller, M.;

Fedosenko, O.; Machulik, S.; Aleksandrova, A.; Monastyrskyi, G. Appl. Opt. 2012, 51, 6789.

(6) Nishida, J.; Fayer, M. D. J. Chem. Phys. 2014, 140, 144702.

(7) Rosenfeld, D. E.; Gengeliczki, Z.; Smith, B. J.; Stack, T. D. P.; Fayer, M. D. Science 2011, 334, 634.

(8) Yan, C.; Yuan, R.; Nishida, J.; Fayer, M. D. J. Phys. Chem. C 2015, 119, 16811.

(9) Rosenfeld, D. E.; Nishida, J.; Yan, C.; Gengeliczki, Z.; Smith, B. J.; Fayer, M. D. J. Phys. Chem. C 2012, 116, 23428.

(10) Kumar, S. K. K.; Tamimi, A.; Fayer, M. D. J. Chem. Phys. 2012, 137, 184201.

(11) Wong, D. B.; Giammanco, C. H.; Fenn, E. E.; Fayer, M. D. J. Phys. Chem. B 2013, 117, 623.

(12) Nishida, J.; Tamimi, A.; Fei, H.; Pullen, S.; Ott, S.; Cohen, S. M.; Fayer, M. D. Proc. Natl. Acad. Sci. 2014, 111, 18442.

(13) Marroux, H. J.; Orr-Ewing, A. J. J. Phys. Chem. B 2016, 120, 4125.

(14) Yan, C.; Yuan, R.; Pfalzgraff, W. C.; Nishida, J.; Wang, L.; Markland, T. E.; Fayer, M. D. Proc. Natl. Acad. Sci. 2016, 201603080.

(15) Wang, C. C.; Pecora, R. J. Chem. Phys. 1980, 72, 5333.

(16) Lipari, G.; Szabo, A. Biophys. J. 1980, 30, 489. 
(17) Gengeliczki, Z.; Rosenfeld, D. E.; Fayer, M. D. J. Chem. Phys. 2010, 132, 244703.

(18) Nienhuys, H.-K.; Bonn, M. J. Phys. Chem. B 2009, 113, 7564.

(19) Rosenfeld, D. E.; Fayer, M. D. J. Chem. Phys. 2012, 137, 064109.

(20) Baumann, J.; Fayer, M. D. J. Chem. Phys. 1986, 85, 4087. 